\title{
Variational and Numerical Analysis of a Static Thermo-Electro-Elastic Problem with Friction
}

\author{
Othman Baiz $\mathbb{D},{ }^{1}$ Hicham Benaissa $\mathbb{D}^{,},{ }^{2}$ Driss El Moutawakil $\mathbb{D},{ }^{1}$ and Rachid Fakhar $\mathbb{D}^{2}$ \\ ${ }^{1}$ Laboratoire MATIC, Université Hassan 1er, 26000 Settat, Morocco \\ ${ }^{2}$ Laboratoire LS3M, Université Hassan 1er, 25000 Khouribga, Morocco \\ Correspondence should be addressed to Rachid Fakhar; rachidfakhar@yahoo.fr
}

Received 12 August 2017; Revised 21 November 2017; Accepted 27 December 2017; Published 28 January 2018

Academic Editor: Francesco Marotti de Sciarra

Copyright (C) 2018 Othman Baiz et al. This is an open access article distributed under the Creative Commons Attribution License, which permits unrestricted use, distribution, and reproduction in any medium, provided the original work is properly cited.

We consider a mathematical model which describes a static frictional contact between a piezoelectric body and a thermally conductive obstacle. The constitutive law is supposed to be thermo-electro-elastic and the contact is modeled with normal compliance and a version of Coulomb's friction law. We derive a variational formulation of the problem and we prove the existence and uniqueness of its solution. The proof is based on some results of elliptic variational inequalities and fixed point arguments. Furthermore, a finite element approximation and a priori error estimates are obtained.

\section{Introduction}

Contact problems involving thermopiezoelectricity arise when there is an interaction between the mechanical, electri$\mathrm{cal}$, and thermal properties of the considered material. This kind of problems has a considerable interest in various fields of modern industries. Indeed, the thermal effects such as thermal deformations and pyroelectric effects are important to many smart ceramic materials. Thus, for some materials, it may be impossible to predict the electromechanical behavior without taking into account the thermal effects; see for example, [1-4]. There is a considerable interest in contact problems involving piezoelectric materials when thermal effects are considered. These so-called thermopiezoelectric materials can operate effectively as distributed sensors and actuators for controlling structural response. In sensor applications, mechanically or thermally induced disturbances can be determined from measurement of the induced electric potential difference (direct piezoelectric effect), whereas in actuator applications deformation or stress can be controlled through the introduction of an appropriate electric potential difference (converse piezoelectric effect). Among the numerous applications of these materials, we cite accelerometers, microphones, ultrasonic transducers, and so on. A good example is the use of this class of materials as sensors and actuators in microelectromechanical systems, for instance, the piezoelectric accelerometer which triggers an airbag in ten thousandths of a second during an accident.

Some general models and their analysis have been established for elastic-piezoelectric materials [5-8], for thermoelastic materials [9-11], and for electro/thermoviscoelastic bodies $[12,13]$. The mathematical treatment of static contact process for thermopiezoelectric materials is recent. The reason lies in the considerable difficulties of the nonlinear evolutionary inequalities modeling the static contact problems present in the variational analysis. Existence and uniqueness results in the study of static contact problems can be found, for instance, in [14-16].

The present paper is a continuation of this kind of models. It deals with a new and nonstandard mathematical model, which is in a form of a system coupling a nonlinear variational inequality for the displacement field and two nonlinear variational equations for the electric potential and the temperature field. The boundary conditions on the contact surface used in this paper is described with a general normal compliance law and the associated Coulomb's friction law, taking into account the electrical and thermal conductivity of the foundation. This work serves two purposes. The first purpose is to provide the variational analysis of the mechanical problems and to show the existence of a unique solution to each model. The 
second is to study a discrete scheme based on finite element method for numerical solutions and to establish the unique solvability of the scheme and derive an optimal order error estimate.

The rest of the paper is structured as follows. In Section 2, we present the model of our frictional thermopiezoelectric contact problem and we derive its variational formulation, given as a coupled system for the displacement field, the electric potential, and the temperature fields. In Section 3, we provide the assumptions on the data and we state the main existence and uniqueness theorem together with its proof. In Section 4, we present the main error estimate results for the finite element approximation of the problem.

\section{The Mathematical Model}

We consider a thermopiezoelectric body that occupies a bounded domain $\Omega$ in $\mathbb{R}^{d}(d=2,3)$ with a Lipschitz continuous boundary $\Gamma$. In the sequel, we decompose $\Gamma$ into three open and disjoint parts $\Gamma_{1}, \Gamma_{2}$, and $\Gamma_{3}$ such that $\Gamma=$ $\bar{\Gamma}_{1} \cup \bar{\Gamma}_{2} \cup \bar{\Gamma}_{3}$ and meas $\left(\Gamma_{1}\right)>0$ on the one hand and we consider a partition of $\bar{\Gamma}_{1} \cup \bar{\Gamma}_{2}$ into two sets $\bar{\Gamma}_{a}$ and $\bar{\Gamma}_{b}$ with disjoint, relatively open sets $\Gamma_{a}$ and $\Gamma_{b}$ such that meas $\left(\Gamma_{a}\right)>0$ on the other hand.

We assume that the body is fixed on $\Gamma_{1}$ where the displacement field vanishes and it is subject to a volume forces of density $f_{0}$, a volume electric charges of density $\phi_{0}$, and a heat source term per unit volume $\vartheta_{0}$ in $\Omega$. We assume also that a surface tractions of density $f_{2}$ and a strength of heat source $\vartheta_{2}$ act on $\Gamma_{2}$ and a surface electric charges of density $\phi_{2}$ acts on $\Gamma_{b}$. Finally, we suppose that the electrical potential vanishes in $\Gamma_{a}$ and the temperature is maintained constant at the part $\Gamma_{1}$ of the boundary, set to be $\theta_{b}$. Over the contact surface $\Gamma_{3}$, the body comes in a frictional contact with a thermally conductive foundation.

Here and below, the indices $i, j, k$, and $l$ run between 1 and $d$, the summation convention over repeated indices is adopted, and the index that follows a comma indicates a partial derivative with respect to the corresponding component of the spatial variable; for example, $u_{i, j}=\partial u_{i} / \partial x_{j}$. In the sequel, let $\mathbb{S}^{d}$ be the space of second order symmetric tensors on $\mathbb{R}^{d}$. The canonical inner products and norms on $\mathbb{R}^{d}$ and $\mathbb{S}^{d}$ are given by

$$
\begin{aligned}
& u \cdot v=u_{i} \cdot v_{i}, \\
& \begin{aligned}
&\|v\|=(v \cdot v)^{1 / 2} \\
& \forall u=\left(u_{i}\right), v=\left(v_{i}\right) \in \mathbb{R}^{d}, \\
& \sigma \cdot \tau=\sigma_{i j} \cdot \tau_{i j}, \\
&\|\tau\|=(\tau \cdot \tau)^{1 / 2} \\
& \forall \sigma=\left(\sigma_{i j}\right), \tau=\left(\tau_{i j}\right) \in \mathbb{S}^{d} .
\end{aligned}
\end{aligned}
$$

To present the mathematical model which describes the physical setting above, we denote by $u, \sigma, \varphi, D, \theta$, and $q$ the displacement field, the stress tensor, the electric potential field, the electric displacement field, the temperature field, and the heat flux vector, respectively. These are functions which depend on the spatial variable $x$. Nevertheless, in what follows we do not indicate explicitly the dependence of these quantities on $x$; that is, we write $\sigma$ instead of $\sigma(x)$. Also, $\varepsilon(u)$ denotes the linearized strain tensor and $E(\varphi)=-\nabla \varphi$ is the electric field.

We assume that the process is static, then the equations of stress equilibrium, the equation of the quasistationary electric field, and the heat conduction equation are

$$
\begin{aligned}
\operatorname{Div} \sigma+f_{0}=0 & \text { in } \Omega, \\
\operatorname{div} D=\phi_{0} & \text { in } \Omega, \\
\operatorname{div} q=\vartheta_{0} & \text { in } \Omega,
\end{aligned}
$$

where $\operatorname{Div} \sigma=\sigma_{i j, j}$ and $\operatorname{div} D=D_{j, j}$ are the divergence operator for tensor and vector field. The material is assumed to be thermopiezoelectric and satisfies the following constitutive laws $[1,4]$ :

$$
\begin{aligned}
& \sigma=\mathfrak{F} \varepsilon(u)-\mathscr{E}^{*} E(\varphi)-\theta \mathscr{M} \quad \text { in } \Omega, \\
& D=\mathscr{E} \varepsilon(u)+\beta E(\varphi)-\theta \mathscr{P} \quad \text { in } \Omega,
\end{aligned}
$$

where $\mathfrak{F}$ is the elasticity tensor, $\mathscr{E}$ is the third-order piezoelectric tensor, $\beta$ represents the electric permittivity tensor, $\mathscr{M}$ is the thermal expansion, and $\mathscr{P}$ denotes the pyroelectric tensor. Moreover, $\mathscr{E}^{*}$ is the transpose of $\mathscr{E}$ and it satisfies

$$
\mathscr{E} \sigma \cdot v=\sigma \cdot \mathscr{E}^{*} v, \quad \forall \sigma \in \mathbb{S}^{d}, v \in \mathbb{R}^{d}
$$

For the heat flux, we adopt the following Fourier-type law:

$$
q=-\mathscr{K} \nabla \theta \text { in } \Omega,
$$

where $\mathscr{K}$ denotes the thermal conductivity tensor. We use classical decomposition in the normal and the tangential components of the displacement $u$ and of the stress $\sigma$ on $\Gamma$; that is,

$$
\begin{aligned}
& u_{v}=u \cdot v, \\
& u_{\tau}=u-u_{\nu} v, \\
& \sigma_{v}=(\sigma v) \cdot v, \\
& \sigma_{\tau}=\sigma v-\sigma_{\nu} \nu,
\end{aligned}
$$

where $v$ is the outward unit normal vector on $\Gamma$ and the physical setting to complete our model with the following boundary conditions:

$$
\begin{array}{cc}
u=0 \quad \text { on } \Gamma_{1}, \\
\sigma v=f_{2} \quad \text { on } \Gamma_{2}, \\
\varphi=0 \quad \text { on } \Gamma_{a}, \\
D \cdot v=\phi_{2} \quad \text { on } \Gamma_{b}, \\
\theta=\theta_{b} \quad \text { on } \Gamma_{1}, \\
q \cdot v=\vartheta_{2} \quad \text { on } \Gamma_{2} .
\end{array}
$$


We model the frictional contact on $\Gamma_{3}$ with the following reduced normal compliance condition:

$$
\begin{aligned}
\sigma_{v} & =-c_{n}\left(u_{v}-g\right)_{+}^{m_{n}} \text { on } \Gamma_{3}, \\
\left\|\sigma_{\tau}\right\| & \leq c_{T}\left(u_{v}-g\right)_{+}^{m_{T}} \\
\left\|\sigma_{\tau}\right\| & <c_{T}\left(u_{v}-g\right)_{+}^{m_{T}} \Longrightarrow u_{\tau}=0 \\
\left\|\sigma_{\tau}\right\| & =c_{T}\left(u_{v}-g\right)_{+}^{m_{T}} \Longrightarrow \sigma_{\tau}=-\lambda u_{\tau}
\end{aligned}
$$

for some $\lambda \geq 0$

on $\Gamma_{3}$,

where $c_{n}, m_{n}, c_{T}$, and $m_{T}$ are material interface parameters and $\left(u_{v}-g\right)_{+}=\max \left(u_{v}-g, 0\right)$ represents the penetration approach; for more detail see [17-19]. Here (15) is the normal compliance power law and (16) is a variant of Coulomb's friction law. Furthermore, the thermoelectric contact is described with the following regularized conditions (see [13, 14]):

$$
\begin{aligned}
& D \cdot v=0 \quad \text { on } \Gamma_{3} \\
& q \cdot v=k_{c}\left(u_{v}-g\right) \phi_{L}\left(\theta-\theta_{F}\right) \quad \text { on } \Gamma_{3},
\end{aligned}
$$

where $k_{c}: r \rightarrow k_{c}(r)$ is the thermal conductance function, supposed to be zero for $r<0$ and positive otherwise, nondecreasing, and Lipschitz continuous, and $\theta_{F}$ is the foundation temperature. The truncate function $\phi_{L}$ is defined by

$$
\phi_{L}(s)= \begin{cases}s & \text { if }|s| \leq L, \\ \frac{s}{|s|} L & \text { if }|s|>L,\end{cases}
$$

where $L$ is a large positive constant. At last, we note that condition (17) describes the fact that the foundation is supposed to be a perfect electrical insulator. Under all these conditions, the classical formulation of our problem is as follows.

Problem $(P)$. Find a displacement field $u: \Omega \rightarrow \mathbb{R}^{d}$, an electric potential $\varphi: \Omega \rightarrow \mathbb{R}$, and a temperature field $\theta$ : $\Omega \rightarrow \mathbb{R}$ such that (2)-(7) and (9)-(18) hold.

Note that once the triplet $(u, \varphi, \theta)$ which solve $(P)$ is known, then the stress tensor $\sigma$, the electric displacement field $D$, and the heat flux $q$ can be obtained from (5) and (7). In order to derive the variational formulation of the problem $P$, we need the following Hilbert spaces:

$$
\begin{aligned}
H & =L^{2}\left(\Omega, \mathbb{R}^{d}\right), \\
\mathscr{H} & =L^{2}\left(\Omega, \mathbb{S}^{d}\right), \\
H_{1} & =H^{1}\left(\Omega, \mathbb{R}^{d}\right) \\
\mathscr{H}_{1} & =\{\sigma \in \mathscr{H} ; \operatorname{Div} \sigma \in H\}, \\
\mathscr{W} & =\left\{D \in H ; \operatorname{div} D \in L^{2}(\Omega)\right\},
\end{aligned}
$$

endowed with the following inner products:

$$
\begin{aligned}
(u, v)_{H} & =\int_{\Omega} u_{i} v_{i} d x, \\
(\sigma, \tau)_{\mathscr{H}} & =\int_{\Omega} \sigma_{i j} \tau_{j i} d x, \\
(u, v)_{H_{1}} & =(u, v)_{H}+(\varepsilon(u), \varepsilon(v))_{\mathscr{H}}, \\
(\sigma, \tau)_{\mathscr{H}_{1}} & =(\sigma, \tau)_{\mathscr{H}}+(\operatorname{Div} \sigma, \operatorname{Div} \tau)_{H}, \\
(D, E)_{\mathscr{W}} & =(D, E)_{H}+(\operatorname{div} D, \operatorname{div} E)_{L^{2}(\Omega)} .
\end{aligned}
$$

Next, we note that, by the Sobolev trace theorem, we can define the trace $\gamma v$ of a function $v \in H_{1}$ on $\Gamma$ such that $\gamma v=v_{\mid \Gamma}$ if $v \in H_{1} \cap C\left(\bar{\Omega}, \mathbb{R}^{d}\right)$. For simplicity, for an element $v \in H_{1}$ we still denote by $v$ its trace $\gamma v$ on $\Gamma$. Let $H_{\Gamma}=H^{1 / 2}\left(\Gamma, \mathbb{R}^{d}\right)$ and $L^{2}(\Gamma)^{d}=L^{2}\left(\Gamma, \mathbb{R}^{d}\right)$, then the trace operator $\gamma: H_{1} \rightarrow H_{\Gamma} \subset$ $L^{2}(\Gamma)^{d}$ is a linear continuous operator; that is, there exists a positive constant $c$, depending only on $\Omega$, such that

$$
\|v\|_{L^{2}(\Gamma)^{d}} \leq c\|v\|_{H_{1}}, \quad \forall v \in H_{1} .
$$

Keeping in mind condition (9), we introduce the closed subspace $V$ of $H_{1}$ given by

$$
V=\left\{v \in H_{1} ; v=0 \text { on } \Gamma_{1}\right\} .
$$

Since meas $\left(\Gamma_{1}\right)>0$, Korn's inequality holds; there exists a positive constant $c_{K}$ which depends only on $\Omega$ and $\Gamma_{1}$ such that (see, e.g., $[7,20]$ )

$$
\|\varepsilon(v)\|_{\mathscr{H}} \geq c_{K}\|v\|_{H_{1}}, \quad \forall v \in V .
$$

We define over the space $V$, the following inner product, and its associated norm:

$$
\begin{aligned}
(u, v)_{V} & =(\varepsilon(u), \varepsilon(v))_{\mathscr{H}}, \\
\|v\|_{V} & =\|\varepsilon(v)\|_{\mathscr{H}} .
\end{aligned}
$$

It comes from (24) that the norms $\|\cdot\|_{V}$ and $\|\cdot\|_{H_{1}}$ are equivalent on $V$ and therefore $\left(V,\|v\|_{V}\right)$ is a real Hilbert space. Finally, note that from (22) and (24), we deduce that there exists a positive constant $c_{0}$ depending on $\Omega, \Gamma_{1}$, and $\Gamma_{3}$ such that

$$
\|v\|_{L^{2}\left(\Gamma_{3}\right)^{d}} \leq \mathcal{c}_{0}\|v\|_{V}, \quad \forall v \in V .
$$

According to the boundary conditions (11) and (13), the electric potential field and the temperature field are, respectively, to be found in the closed subspace $W$ and $Q$ of $H^{1}(\Omega)$ given by

$$
\begin{aligned}
& W=\left\{\psi \in H^{1}(\Omega) ; \psi=0 \text { on } \Gamma_{a}\right\}, \\
& Q=\left\{\theta \in H^{1}(\Omega) ; \theta=0 \text { on } \Gamma_{1}\right\} .
\end{aligned}
$$

Since meas $\left(\Gamma_{a}\right)>0$, the Friedrichs-Poincaré inequality holds and thus there exists a positive constant $c_{F}$ depending only on $\Omega$ and $\Gamma_{a}$ such that

$$
\|\nabla \psi\|_{H} \geq c_{F}\|\psi\|_{H^{1}(\Omega)} \quad \forall \psi \in W .
$$


We introduce on $W$ the inner product $(\varphi, \psi)_{W}=(\nabla \varphi, \nabla \psi)_{H}$ and its associated norm $\|\psi\|_{W}$. It follows from (28) that the norms $\|\cdot\|_{H^{1}(\Omega)}$ and $\|\cdot\|_{W}$ are equivalent on $W$ and therefore, the space $\left(W,\|\cdot\|_{W}\right)$ is a real Hilbert. Since meas $\left(\Gamma_{1}\right)>0$, we can prove in an analogous way that the norm $\|\cdot\|_{Q}$ associated with the inner product $(\theta, \xi)_{\mathrm{Q}}=(\nabla \theta, \nabla \xi)_{H}$ is equivalent to the usual norm $\|\cdot\|_{H^{1}(\Omega)}$ on $Q$ and thus, $\left(Q,\|\cdot\|_{Q}\right)$ is a real Hilbert space. Using the Sobolev trace theorem, we get that there exists a positive constant $c_{1}$ depending only on $\Omega, \Gamma_{a}$, and $\Gamma_{3}$ such that

$$
\|\psi\|_{L^{2}\left(\Gamma_{3}\right)} \leq c_{1}\|\psi\|_{W} \quad \forall \psi \in W
$$

and a positive constant $c_{2}$ which depends only on $\Omega, \Gamma_{1}$, and $\Gamma_{3}$ such that

$$
\|\xi\|_{L^{2}\left(\Gamma_{3}\right)} \leq c_{2}\|\xi\|_{Q} \quad \forall \xi \in Q
$$

In the study of the mechanical problem $(P)$, the following assumptions will be needed:

$\left(\mathrm{h}_{1}\right)$ The elasticity operator $\mathfrak{F}=\left(f_{i j k l}\right): \Omega \times \mathbb{S}^{d} \rightarrow \mathbb{S}^{d}$, the electric permittivity tensor $\beta=\left(\beta_{i j}\right): \Omega \times \mathbb{R}^{d} \rightarrow \mathbb{R}^{d}$, and the thermal conductivity tensor $\mathscr{K}=\left(k_{i j}\right): \Omega \times$ $\mathbb{R}^{d} \rightarrow \mathbb{R}^{d}$ satisfy the usual properties of symmetry, boundedness, and ellipticity

$$
\begin{aligned}
f_{i j k l} & =f_{j i k l}=f_{k l i j} \in L^{\infty}(\Omega), \\
\beta_{i j} & =\beta_{j i} \in L^{\infty}(\Omega), \\
k_{i j} & =k_{j i} \in L^{\infty}(\Omega)
\end{aligned}
$$

and there exist positive constants $m_{\mathfrak{F}}, m_{\beta}$, and $m_{\mathscr{K}}$ such that

$$
\begin{aligned}
& f_{i j k l} \xi_{i j} \xi_{k l} \geq m_{\mathscr{F}}\|\xi\|^{2} \quad \forall \xi=\left(\xi_{i j}\right) \in \mathbb{S}^{d}, \\
& \beta_{i j} \zeta_{i} \zeta_{j} \geq m_{\beta}\|\zeta\|^{2} \\
& k_{i j} \zeta_{i} \zeta_{j} \geq m_{\mathscr{K}}\|\zeta\|^{2} \\
& \forall \zeta=\left(\zeta_{i}\right) \in \mathbb{R}^{d} .
\end{aligned}
$$

$\left(\mathrm{h}_{2}\right)$ The piezoelectric tensor $\mathscr{E}=\left(e_{i j k}\right): \Omega \times \mathbb{S}^{d} \rightarrow \mathbb{R}^{d}$, the thermal expansion tensor $\mathscr{M}=\left(m_{i j}\right): \Omega \times \mathbb{R}^{d} \rightarrow$ $\mathbb{R}^{d}$, and the pyroelectric tensor $\mathscr{P}=\left(p_{i}\right): \Omega \rightarrow \mathbb{R}^{d}$ satisfy

$$
\begin{gathered}
e_{i j k}=e_{i k j} \in L^{\infty}(\Omega), \\
m_{i j}=m_{j i} \in L^{\infty}(\Omega), \\
p_{i} \in L^{\infty}(\Omega) .
\end{gathered}
$$

$\left(h_{3}\right)$ The thermal conductance $k_{c}: \Gamma_{3} \times \mathbb{R} \rightarrow \mathbb{R}_{+}$satisfies the following conditions:

$\left|k_{c}(x, u)\right| \leq M_{k}$

$\forall u \in \mathbb{R}, x \in \Gamma_{3}$ where $M_{k}$ is a positive constant, $x \longmapsto k_{c}(x, u)$ is mesurable on $\Gamma_{3}$

$\forall u \in \mathbb{R}$ and is zero $\forall u \leq 0$.

$\left(\mathrm{h}_{4}\right)$ The function $u \mapsto k_{c}(x, u)$ is a Lipschitz function on $\mathbb{R}$ for all $x \in \Gamma_{3}$; that is,

$\left|k_{c}\left(x, u_{1}\right)-k_{c}\left(x, u_{2}\right)\right| \leq L_{k}\left|u_{1}-u_{2}\right|$ $\forall u_{1}, u_{2} \in \mathbb{R}$ with $L_{k}>0$ is a constant.

$\left(h_{5}\right)$ The forces, the traction, the charges densities, and the strength of the heat source satisfy

$$
\begin{aligned}
& f_{0} \in L^{2}(\Omega)^{d}, \\
& f_{2} \in L^{2}\left(\Gamma_{2}\right)^{d}, \\
& \phi_{0} \in L^{2}(\Omega), \\
& \phi_{2} \in L^{2}\left(\Gamma_{b}\right), \\
& \vartheta_{0} \in L^{2}(\Omega), \\
& \vartheta_{2} \in L^{2}\left(\Gamma_{2}\right) .
\end{aligned}
$$

$\left(\mathrm{h}_{6}\right)$ The foundation temperature satisfies

$$
\theta_{F} \in L^{2}\left(\Gamma_{3}\right)
$$

$\left(h_{7}\right)$ The friction bound function and the coefficient of friction satisfy

$$
c_{n}, c_{T} \in L^{\infty}\left(\Gamma_{3}\right), \quad c_{n}, c_{T} \geq 0
$$

$\left(h_{8}\right)$ The material interface parameters satisfy

$$
\begin{aligned}
& 1 \leq m_{n}, m_{T}<\infty \quad \text { if } d=2, \\
& 1 \leq m_{n}, m_{T} \leq 2 \quad \text { if } d=3 .
\end{aligned}
$$

Using the Riesz representation theorem, we can define $f \in V$, $\phi \in W$, and $\vartheta \in Q$ as follows:

$$
\begin{aligned}
& (f, v)_{V}=\int_{\Omega} f_{0} \cdot v d x+\int_{\Gamma_{2}} f_{2} \cdot v d a, \quad \forall v \in V, \\
& (\phi, \xi)_{W}=\int_{\Omega} \phi_{0} \xi d x-\int_{\Gamma_{b}} \phi_{2} \xi d a, \quad \forall \xi \in W, \\
& (\vartheta, \eta)_{Q}=\int_{\Omega} \vartheta_{0} \eta d x+\int_{\Gamma_{2}} \vartheta_{2} \eta d a, \quad \forall \eta \in Q .
\end{aligned}
$$


We consider the functionals $j_{n}, j_{T}: V \times V \rightarrow \mathbb{R}$ and $\chi:$ $V \times Q \times Q \rightarrow \mathbb{R}$ defined by

$$
\begin{aligned}
j_{n}(u, v) & =\int_{\Gamma_{3}} c_{n}\left(u_{v}-g\right)_{+}^{m_{n}} v_{v} d a, \quad \forall u, v \in V, \\
j_{T}(u, v) & =\int_{\Gamma_{3}} c_{T}\left(u_{v}-g\right)_{+}^{m_{T}}\left|v_{\tau}\right| d a, \quad \forall u, v \in V, \\
\chi(u, \theta, \eta) & =\int_{\Gamma_{3}} k_{c}\left(u_{v}-g\right) \phi_{L}\left(\theta-\theta_{F}\right) \eta d a,
\end{aligned}
$$

$\forall u \in V, \forall \theta, \eta \in Q$.

It follows from $\left(h_{3}\right)$ and $\left(h_{5}\right)-\left(h_{8}\right)$ that the integrals above are well defined. We note that if $\sigma \in \mathscr{H}_{1}$ and $\Psi \in \mathscr{W}$ are sufficiently regular, the following Greens formulas hold:

$$
\begin{aligned}
& \int_{\Gamma} \sigma v \cdot v d a=(\sigma, \varepsilon(v))_{\mathscr{C}}+(\operatorname{Div} \sigma, v)_{H}, \quad \forall v \in H_{1}, \\
& \int_{\Gamma} \Psi \cdot v \psi d a=(\Psi, \nabla \psi)_{H}+(\operatorname{div} \Psi, v)_{L^{2}(\Omega)},
\end{aligned}
$$

$\forall v \in H^{1}(\Omega)$.

Using the previous Greens formulas, it is straightforward to see that if $(u, \varphi, \theta)$ are sufficiently regular functions which satisfy (2)-(7) and (9)-(18), then

$$
\begin{aligned}
& (\sigma, \varepsilon(v)-\varepsilon(u))_{\mathscr{C}}+j_{n}(u, v-u)+j_{T}(u, v) \\
& \quad-j_{T}(u, u) \geq(f, v-u)_{V}, \\
& (D, \nabla \xi)_{H}+(\phi, \xi)_{W}=0, \\
& (q, \nabla \eta)_{H}=\chi(u, \theta, \eta)-(\vartheta, \eta)_{Q},
\end{aligned}
$$

for all $v \in V, \xi \in W$, and $\eta \in Q$. We use (5), (7), and the notation $E(\varphi)=-\nabla \varphi$ to obtain the following variational formulation of our problem.

Problem $(P V)$. Find a displacement field $u \in V$ an electric potential $\varphi \in W$ and a temperature field $\theta \in Q$ such that

$$
\begin{aligned}
& \left(\mathfrak{F}_{\varepsilon}(u), \varepsilon(v)-\varepsilon(u)\right)_{\mathscr{H}}+\left(\mathscr{E}^{*} \nabla \varphi, \varepsilon(v)-\varepsilon(u)\right)_{\mathscr{H}} \\
& -(\mathscr{M} \theta, \varepsilon(v)-\varepsilon(u))_{\mathscr{H}} j_{n}(u, v-u)+j_{T}(u, v) \\
& -j_{T}(u, u) \geq(f, v-u)_{V}, \quad \forall v \in V, \\
& (\beta \nabla \varphi, \nabla \xi)_{H}-(\mathscr{E} \varepsilon(u), \nabla \xi)_{H} \\
& -(\mathscr{P} \theta, \nabla \xi)_{H}=(\phi, \xi)_{W}, \quad \forall \xi \in W, \\
& (\mathscr{K} \nabla \theta, \nabla \eta)_{H}+\chi(u, \theta, \eta)=(\vartheta, \eta)_{Q}, \quad \forall \eta \in Q .
\end{aligned}
$$

\section{Existence and Uniqueness of Weak Solution}

The main existence and uniqueness result in the study of the problem $(P V)$ are as follows.
Theorem 1. Assume $\left(h_{1}\right)-\left(h_{3}\right)$ and $\left(h_{5}\right)-\left(h_{8}\right)$ hold. Then one has the following:

(1) The problem (PV) has at least one solution.

(2) Under $\left(h_{4}\right)$, there exists a constant $L^{*}>0$ such that if

$$
m_{T}+\|\mathscr{P}\|+\|\mathscr{M}\|+L_{k} L+M_{k}<L^{*},
$$

then the problem $(P V)$ has a unique solution.

Here the norms of the tensors $\mathscr{P}=\left(p_{i}\right)$ and $\mathscr{M}=\left(m_{i j}\right)$ are given by

$$
\begin{aligned}
\|\mathscr{P}\| & =\max _{1 \leqslant i \leqslant d} p_{i}, \\
\|\mathscr{M}\| & =\max _{1 \leqslant i, j \leqslant d} m_{i j} .
\end{aligned}
$$

The proof will be carried out in several steps and it is based on arguments of variational inequalities and fixed point techniques. We assume in what follows that $\left(\mathrm{h}_{1}\right)-\left(\mathrm{h}_{3}\right)$ and $\left(\mathrm{h}_{5}\right)-\left(\mathrm{h}_{8}\right)$ hold and for every $z=\left(z_{1}, z_{2}\right)$ where $z_{1}, z_{2} \geq 0 \in$ $L^{2}\left(\Gamma_{3}\right)$, we define

$$
\begin{aligned}
& \chi_{1}(z, \eta)=\int_{\Gamma_{3}} z_{1} \eta d a, \quad \forall \eta \in Q, \\
& \chi_{2}(z, v)=\int_{\Gamma_{3}} z_{2}\left|v_{\tau}\right| d a, \quad \forall v \in V .
\end{aligned}
$$

For all $z$ supposed to be known, we consider the following auxiliary problem.

$\operatorname{Problem}\left(P V_{z}\right)$. Find the elements $u_{z} \in V, \varphi_{z} \in W$, and $\theta_{z} \in Q$ such that

$$
\begin{aligned}
& \quad\left(\mathscr{F}_{\varepsilon}\left(u_{z}\right), \varepsilon(v)-\varepsilon\left(u_{z}\right)\right)_{\mathscr{C}} \\
& \quad+\left(\mathscr{E}^{*} \nabla \varphi_{z}, \varepsilon(v)-\varepsilon\left(u_{z}\right)\right)_{\mathscr{C}} \\
& \quad-\left(\mathscr{M} \theta_{z}, \varepsilon(v)-\varepsilon\left(u_{z}\right)\right)_{\mathscr{H}} j_{n}\left(u_{z}, v-u_{z}\right)+\chi_{2}(z, v) \\
& \quad-\chi_{2}\left(z, u_{z}\right) \geq\left(f, v-u_{z}\right)_{V}, \quad \forall v \in V, \\
& \left(\beta \nabla \varphi_{z}, \nabla \xi\right)_{H}-\left(\mathscr{E} \varepsilon\left(u_{z}\right), \nabla \xi\right)_{H} \\
& \quad-\left(\mathscr{P} \theta_{z}, \nabla \xi\right)_{H}=(\phi, \xi)_{W}, \quad \forall \xi \in W, \\
& \left(\mathscr{K} \nabla \theta_{z}, \nabla \eta\right)_{H}+\chi_{1}(z, \eta)=(\vartheta, \eta)_{Q}, \quad \forall \eta \in Q .
\end{aligned}
$$

In the study of this problem, the two following results will be needed.

Lemma 2. Let $\alpha$ be a positive real number. For all $x, y \in \mathbb{R}$, one has

$$
\left((x)_{+}^{\alpha}-(y)_{+}^{\alpha}\right)(x-y) \geq 0
$$

and consequently for all $g \in \mathbb{R}$, one has

$$
\left((x-g)_{+}^{\alpha}-(y-g)_{+}^{\alpha}\right)(x-y) \geq 0, \quad \forall x, y \in \mathbb{R} .
$$

Proof. This lemma can be obtained by examining the two cases $x y \geq 0$ and $x y \leq 0$. 
Theorem 3. Let $\Omega$ be open bounded set of $\mathbb{R}^{d}$ with a Lipschitz boundary $\Gamma$ and $1<p<\infty$. The trace operator $\gamma: W^{1, p}(\Omega) \rightarrow$ $L^{r}(\Gamma)$ satisfies the following results:

(1) If $p<d$, the map $\gamma$ is compact for any $1 \leq r<(d p-$ $p) /(d-p)$. Then, there exists $N_{r}>0$ such that

$$
\|v\|_{L^{r}(\Gamma)} \leq N_{r}\|v\|_{W^{1, p}(\Omega)} \quad \forall v \in W^{1, p}(\Omega) .
$$

(2) If $p \geq d$, the map $\gamma$ is compact for any $r \geq 1$ and then, there exists $N_{r}>0$ such that

$$
\|v\|_{L^{r}(\Gamma)} \leq N_{r}\|v\|_{W^{1, p}(\Omega)} \quad \forall v \in W^{1, p}(\Omega) .
$$

Proof. For the proof of the trace Theorem 3, we can refer to $[21,22]$.

Lemma 4. Under the assumptions $\left(h_{1}\right)-\left(h_{3}\right)$ and $\left(h_{5}\right)-\left(h_{8}\right)$, the problem $\left(P V_{z}\right)$ has a unique solution $\left(u_{z}, \varphi_{z}, \theta_{z}\right) \in V \times$ $W \times Q$ which depends Lipschitz continuously on $z$.

Proof. We use Riesz's representation theorem to define the element $\vartheta_{z}$ of $Q$

$$
\left(\vartheta_{z}, \eta\right)_{\mathrm{Q}}=(\vartheta, \eta)_{\mathrm{Q}}-\chi_{1}(z, \eta) \quad \forall \eta \in \mathrm{Q}
$$

and the operator $\mathscr{T}: Q \rightarrow Q$ such that

$$
\left(\mathscr{T} \theta_{z}, \eta\right)_{\mathrm{Q}}=\left(\mathscr{K} \nabla \theta_{z}, \nabla \eta\right)_{L^{2}(\Omega)^{d}}, \quad \forall \eta \in Q .
$$

Thus equation (55) will be

Find $\theta_{z} \in Q$ such that $\left(\mathscr{T} \theta_{z}, \eta\right)_{\mathrm{Q}}=\left(\vartheta_{z}, \eta\right)_{\mathrm{Q}}$,

$$
\forall \eta \in Q \text {. }
$$

Using the assumptions of $\mathscr{K}\left(\right.$ see $\left.\left(\mathrm{h}_{1}\right)\right)$, we can deduce that $\mathscr{T}$ is a linear symmetric and positive definite operator. Hence, $\mathscr{T}$ is linear continuous and invertible operator on $Q$ and let $\mathscr{C}$ denote its inverse. Thus, by the Lax-Milgram theorem, we get that problem (62) has a unique solution

$$
\theta_{z}=\mathscr{C} \vartheta_{z} \in Q .
$$

It follows from the properties of the operators $\mathscr{T}, \mathscr{M}$, and $\mathscr{P}$ that $\mathscr{M C}$ and $\mathscr{P} \mathscr{C}$ are linear continuous operators. Moreover, we apply Riesz's representation theorem to define the element $\phi_{z} \in W$ and the operators $B: W \rightarrow W$ and $C: V \rightarrow W$ as follows:

$$
\begin{aligned}
& \left(\phi_{z}, \xi\right)_{W}=(\phi, \xi)_{W}+\left(\mathscr{P} \mathscr{C} \vartheta_{z}, \nabla \xi\right)_{H}, \quad \forall \xi \in W, \\
& (B \varphi, \xi)_{W}=(\beta \nabla \varphi, \nabla \xi)_{H}, \quad \forall(\varphi, \xi) \in W \times W, \\
& (C v, \xi)_{W}=(\mathscr{E} \varepsilon(v), \nabla \xi)_{H}, \quad \forall(v, \xi) \in V \times W .
\end{aligned}
$$

Let $C^{*}$ be the adjoint operator of $C$ and then, it comes from (66) that

$$
\left(C^{*} \xi, v\right)_{W}=\left(\mathscr{E}^{*} \nabla \xi, \varepsilon(v)\right)_{H} \quad \forall v \in V \xi \in W .
$$

Next, we replace (64), (65), and (66) in (54) to obtain

$$
B \varphi_{z}=\phi_{z}+C u_{z}
$$

Keeping in mind the properties of $\beta\left(\right.$ see $\left.\left(\mathrm{h}_{1}\right)\right)$, we get that $B$ is a linear symmetric and positive definite operator. Hence, the operator $B$ is invertible and let $B^{-1}$ be its inverse. We have

$$
\varphi_{z}=B^{-1} C u_{z}+B^{-1} \phi_{z} \in W .
$$

It comes from (63), (69) that inequality (53) is equivalent to find $u_{z} \in V$ such that

$$
\begin{gathered}
\left(\mathfrak{F}_{\varepsilon}\left(u_{z}\right), \varepsilon(v)-\varepsilon\left(u_{z}\right)\right)_{\mathscr{C}}+\left(C^{*} B^{-1} C u_{z}, v-u_{z}\right)_{V} \\
\quad+j_{n}\left(u_{z}, v-u_{z}\right)+\chi_{2}(z, v)-\chi_{2}\left(z, u_{z}\right) \\
\geq\left(f, v-u_{z}\right)_{V}+\left(M \mathscr{C} \vartheta_{z}, \varepsilon(v)-\varepsilon(u)\right)_{\mathscr{C}} \\
+\left(C^{*} B^{-1} \phi_{z}, v-u_{z}\right)_{V} \quad \forall v \in V .
\end{gathered}
$$

The variational problem above is equivalent to the following minimization problem:

$$
\text { Find } u_{z} \in V \text { such that } J_{z}\left(u_{z}\right)=\inf _{v \in V} J_{z}(v),
$$

where the functional $J_{z}: V \rightarrow \mathbb{R}$ is defined as follows:

$$
\begin{aligned}
J_{z}(v)= & \frac{1}{2}(\mathfrak{F} \varepsilon(v), \varepsilon(v))_{\mathscr{H}}+\frac{1}{2}\left(C^{*} B^{-1} C v, v\right)_{V} \\
& +\int_{\Gamma_{3}} \frac{c_{n}}{m_{n}+1}\left(v_{v}-g\right)_{+}^{m_{n}+1} d a+\chi_{2}(z, v) \\
& -(f, v)_{V}-\left(\mathscr{M} \mathscr{C} \vartheta_{z}, \mathcal{\varepsilon}(v)\right)_{\mathscr{H}} \\
& -\left(C^{*} B^{-1} \phi_{z}, v\right)_{V}, \quad \forall v \in V .
\end{aligned}
$$

We consider the function $\tilde{j}_{n}(v)=\int_{\Gamma_{3}}\left(c_{n} /\left(m_{n}+1\right)\right)\left(v_{v}-\right.$ $g)_{+}^{m_{n}+1} d a$ whose derivative is

$$
\left\langle D \tilde{j}_{n}(v), w\right\rangle=\int_{\Gamma_{3}} c_{n}\left(v_{v}-g\right)_{+}^{m_{n}} w_{n} d a, \quad \forall w \in V .
$$

Applying Lemma 2, we get for all $v$ and $w$ of $V$ that

$$
\begin{aligned}
& \left\langle D \tilde{j}_{n}(v)-D \tilde{j}_{n}(w), v-w\right\rangle \\
& \quad=\int_{\Gamma_{3}} c_{n}\left[\left(v_{\nu}-g\right)_{+}^{m_{n}}-\left(w_{\nu}-g\right)_{+}^{m_{n}}\right]\left(v_{\nu}-w_{\nu}\right) d a \\
& \quad \geq 0 .
\end{aligned}
$$

Hence the function $\tilde{j}_{n}$ is convex. In plus, it follows from the strict convexity of $J_{z}-\tilde{j}_{n}$ that the functional $J_{z}=\left(J_{z}-\right.$ $\left.\tilde{j}_{n}\right)+\tilde{j}_{n}$ is strictly convex on $V$. Moreover, since $J_{z}-\tilde{j}_{n}$ is coercive and $J_{z}(v) \geq J_{z}(v)-\tilde{j}_{n}(v)$, we deduce that $J_{z}$ is coercive. Consequently, the minimization problem has a unique solution $u_{z} \in V$. Therefore, keeping in mind (63) and (69), we conclude that the variational problem $\left(P V_{z}\right)$ has a unique solution $\left(u_{z}, \varphi_{z}, \theta_{z}\right)$ of $V \times W \times Q$. 
For the second part of Lemma 4, let $z=\left(z_{1}, z_{2}\right)$ and $z^{\prime}=\left(z_{1}^{\prime}, z_{2}^{\prime}\right)$ be two given elements of the reflexive space $L^{2}\left(\Gamma_{3}\right)^{2}$ such that $z_{2} \geq 0$ and $z_{2}^{\prime} \geq 0$. We consider $\left(u_{z}, \varphi_{z}, \theta_{z}\right)$, $\left(u_{z^{\prime}}, \varphi_{z^{\prime}}, \theta_{z^{\prime}}\right)$, the unique solution of the problems $\left(P V_{z}\right)$, $\left(P V_{z^{\prime}}\right)$, respectively. Then, the variational inequality (53) leads to

$$
\begin{aligned}
& \left(\mathfrak{F}_{\varepsilon}\left(u_{z}\right), \varepsilon(v)-\varepsilon\left(u_{z}\right)\right)_{\mathscr{H}}+\left(\mathscr{E}^{*} \nabla \varphi_{z}, \varepsilon(v)-\varepsilon\left(u_{z}\right)\right)_{\mathscr{H}} \\
& \quad-\left(\mathscr{M} \theta_{z}, \varepsilon(v)-\varepsilon\left(u_{z}\right)\right)_{\mathscr{H}}+j_{n}\left(u_{z}, v-u_{z}\right) \\
& \quad+\chi_{2}(z, v)-\chi_{2}\left(z, u_{z}\right) \geq\left(f, v-u_{z}\right)_{V}, \quad \forall v \in V, \\
& \left(\mathfrak{F}_{\varepsilon}\left(u_{z^{\prime}}\right), \varepsilon(v)-\varepsilon\left(u_{z^{\prime}}\right)\right)_{\mathscr{H}} \\
& \quad+\left(\mathscr{E}^{*} \nabla \varphi_{z^{\prime}}, \varepsilon(v)-\varepsilon\left(u_{z^{\prime}}\right)\right)_{\mathscr{H}} \\
& \quad-\left(\mathscr{M} \theta_{z^{\prime}}, \varepsilon(v)-\varepsilon\left(u_{z^{\prime}}\right)\right)_{\mathscr{H}}+j_{n}\left(u_{z^{\prime}}, v-u_{z^{\prime}}\right) \\
& \quad+\chi_{2}\left(z^{\prime}, v\right)-\chi_{2}\left(z^{\prime}, u_{z^{\prime}}\right) \geq\left(f, v-u_{z^{\prime}}\right)_{V},
\end{aligned}
$$

$\forall v \in V$.

We take $v=u_{z^{\prime}}$ in the first inequality and $v=u_{z}$ in the second to get

$$
\begin{aligned}
& \left(\Im_{\varepsilon}\left(u_{z}\right), \varepsilon\left(u_{z^{\prime}}\right)-\varepsilon\left(u_{z}\right)\right)_{\mathscr{H}} \\
& \quad+\left(\mathscr{E}^{*} \nabla \varphi_{z}, \varepsilon\left(u_{z^{\prime}}\right)-\varepsilon\left(u_{z}\right)\right)_{\mathscr{H}} \\
& \quad-\left(\mathscr{M} \theta_{z}, \varepsilon\left(u_{z^{\prime}}\right)-\varepsilon\left(u_{z}\right)\right)_{\mathscr{H}}+j_{n}\left(u_{z}, u_{z^{\prime}}-u_{z}\right) \\
& \quad+\chi_{2}\left(z, u_{z^{\prime}}\right)-\chi_{2}\left(z, u_{z}\right) \geq\left(f, u_{z^{\prime}}-u_{z}\right)_{V}, \\
& \left(\mho \varepsilon\left(u_{z^{\prime}}\right), \varepsilon\left(u_{z}\right)-\varepsilon\left(u_{z^{\prime}}\right)\right)_{\mathscr{H}} \\
& \quad+\left(\mathscr{E}^{*} \nabla \varphi_{z^{\prime}}, \varepsilon\left(u_{z}\right)-\varepsilon\left(u_{z^{\prime}}\right)\right)_{\mathscr{H}} \\
& \quad-\left(\mathscr{M} \theta_{z^{\prime}}, \varepsilon\left(u_{z}\right)-\varepsilon\left(u_{z^{\prime}}\right)\right)_{\mathscr{H}}+j_{n}\left(u_{z^{\prime}}, u_{z}-u_{z^{\prime}}\right) \\
& \quad+\chi_{2}\left(z^{\prime}, u_{z}\right)-\chi_{2}\left(z^{\prime}, u_{z^{\prime}}\right) \geq\left(f, u_{z}-u_{z^{\prime}}\right)_{V}
\end{aligned}
$$

and by adding the two induced inequalities, we obtain

$$
\begin{aligned}
(\mathfrak{F} \varepsilon & \left.\left(u_{z}\right)-\mathfrak{F} \varepsilon\left(u_{z^{\prime}}\right), \varepsilon\left(u_{z}\right)-\varepsilon\left(u_{z^{\prime}}\right)\right)_{\mathscr{H}} \\
& +\left(\mathscr{E}^{*} \nabla \varphi_{z}-\mathscr{E}^{*} \nabla \varphi_{z^{\prime}}, \varepsilon\left(u_{z}\right)-\varepsilon\left(u_{z^{\prime}}\right)\right)_{\mathscr{H}} \\
\leq & \chi_{2}\left(z, u_{z}\right)-\chi_{2}\left(z, u_{z^{\prime}}\right)+\chi_{2}\left(z^{\prime}, u_{z^{\prime}}\right) \\
& -\chi_{2}\left(z^{\prime}, u_{z}\right)+j_{n}\left(u_{z^{\prime}}, u_{z}-u_{z^{\prime}}\right) \\
& -j_{n}\left(u_{z}, u_{z}-u_{z^{\prime}}\right) \\
& +\left(\mathscr{M} \theta_{z}-\mathscr{M} \theta_{z^{\prime}}, \varepsilon\left(u_{z}\right)-\varepsilon\left(u_{z^{\prime}}\right)\right)_{\mathscr{H}} .
\end{aligned}
$$

Moreover, the definition of $j_{n}$ and Lemma 2 imply that

$$
\begin{aligned}
& j_{n}\left(u_{z^{\prime}}, u_{z}-u_{z^{\prime}}\right)-j_{n}\left(u_{z}, u_{z}-u_{z^{\prime}}\right) \\
&=-\int_{\Gamma_{3}} c_{n}\left[\left(u_{z^{\prime} v}-g\right)_{+}^{m_{n}}-\left(u_{z v}-g\right)_{+}^{m_{n}}\right] \\
& \cdot\left(u_{z^{\prime} v}-u_{z v}\right) d a \leq 0 .
\end{aligned}
$$

Then, we have

$$
\begin{aligned}
(\mathfrak{F} \varepsilon & \left.\left(u_{z}\right)-\mathfrak{\mho} \varepsilon\left(u_{z^{\prime}}\right), \varepsilon\left(u_{z}\right)-\varepsilon\left(u_{z^{\prime}}\right)\right)_{\mathscr{H}} \\
& +\left(\mathscr{E}^{*} \nabla \varphi_{z}-\mathscr{E}^{*} \nabla \varphi_{z^{\prime}}, \varepsilon\left(u_{z}\right)-\varepsilon\left(u_{z^{\prime}}\right)\right)_{\mathscr{H}} \\
\leq & \chi_{2}\left(z, u_{z}\right)-\chi_{2}\left(z, u_{z^{\prime}}\right)+\chi_{2}\left(z^{\prime}, u_{z^{\prime}}\right) \\
& -\chi_{2}\left(z^{\prime}, u_{z}\right) \\
& +\left(\mathscr{M} \theta_{z}-\mathscr{M} \theta_{z^{\prime}}, \varepsilon\left(u_{z}\right)-\varepsilon\left(u_{z^{\prime}}\right)\right)_{\mathscr{H}} .
\end{aligned}
$$

It follows from (26) and the definition of $\chi_{2}$ that

$$
\begin{aligned}
& \chi_{2}\left(z, u_{z}\right)-\chi_{2}\left(z, u_{z^{\prime}}\right)+\chi_{2}\left(z^{\prime}, u_{z^{\prime}}\right)-\chi_{2}\left(z^{\prime}, u_{z}\right) \\
& \quad=\int_{\Gamma_{3}}\left(\left|z_{1}\right|-\left|z_{1}^{\prime}\right|\right)\left(u_{z_{\tau}}-u_{z_{\tau}^{\prime}}\right) d a \\
& \quad \leq c_{0}\left\|z_{1}-z_{1}^{\prime}\right\|_{L^{2}\left(\Gamma_{3}\right)}\left\|u_{z}-u_{z^{\prime}}\right\|_{V} .
\end{aligned}
$$

In addition, the variational equation (54) leads to

$$
\begin{aligned}
& \quad\left(\beta \nabla \varphi_{z}, \nabla \xi\right)_{H}-\left(\mathscr{E} \varepsilon\left(u_{z}\right), \nabla \xi\right)_{H}-\left(\mathscr{P} \theta_{z}, \nabla \xi\right)_{H} \\
& \quad=(\phi, \xi)_{W}, \\
& \left(\beta \nabla \varphi_{z^{\prime}}, \nabla \xi\right)_{H}-\left(\mathscr{E} \varepsilon\left(u_{z^{\prime}}\right), \nabla \xi\right)_{H}-\left(\mathscr{P} \theta_{z^{\prime}}, \nabla \xi\right)_{H} \\
& \quad=(\phi, \xi)_{W} .
\end{aligned}
$$

After taking $\xi=\varphi_{z}-\varphi_{z^{\prime}}$ in (81) and $\xi=\varphi_{z^{\prime}}-\varphi_{z}$ in (81), we add the induced equations

$$
\begin{aligned}
( & \left.\beta \nabla \varphi_{z}-\nabla \varphi_{z^{\prime}}, \nabla \varphi_{z}-\nabla \varphi_{z^{\prime}}\right)_{H} \\
& \quad-\left(\mathscr{E} \varepsilon\left(u_{z}\right)-\mathscr{E} \varepsilon\left(u_{z^{\prime}}\right), \nabla \varphi_{z}-\nabla \varphi_{z^{\prime}}\right)_{H} \\
= & \left(\mathscr{P} \theta_{z}-\mathscr{P} \theta_{z^{\prime}}, \nabla \varphi_{z}-\nabla \varphi_{z^{\prime}}\right)_{H} .
\end{aligned}
$$

Using (79), (80), (83), (30), and (6), the strong monotonicity of $\mathfrak{F}$, and the ellipticity of $\beta$ and after some algebra, we find that there exists a constant $c_{b}>0$ such that

$$
\begin{aligned}
& \left\|u_{z}-u_{z^{\prime}}\right\|_{V}+\left\|\varphi_{z}-\varphi_{z^{\prime}}\right\|_{W} \\
& \quad \leq c_{b}\left(\left\|z_{1}-z_{1}^{\prime}\right\|_{L^{2}\left(\Gamma_{3}\right)}+\left\|\theta_{z}-\theta_{z^{\prime}}\right\|_{Q}\right) .
\end{aligned}
$$

From (60), (51), and (30), we obtain

$$
\begin{aligned}
\left(\vartheta_{z^{\prime}}-\vartheta_{z}, \eta\right)_{\mathrm{Q}} & =\left(\chi_{1}\left(z^{\prime}, \eta\right)-\chi_{1}(z, \eta)\right) \\
& \leq c_{2}\left\|z_{1}^{\prime}-z_{1}\right\|_{L^{2}\left(\Gamma_{3}\right)}\|\eta\|_{\mathrm{Q}},
\end{aligned}
$$

and from (61), (62), (85), and the ellipticity of $\mathscr{K}$, we find that there exists $c_{k}>0$ such that

$$
\left\|\theta_{z}-\theta_{z^{\prime}}\right\|_{\mathrm{Q}} \leq c_{k}\left\|z_{2}-z_{2}^{\prime}\right\|_{L^{2}\left(\Gamma_{3}\right)} .
$$

Finally, we combine (84) and (87) to find that there exists a constant $c_{3}>0$ such that

$$
\begin{gathered}
\left\|u_{z}-u_{z^{\prime}}\right\|_{V}+\left\|\varphi_{z}-\varphi_{z^{\prime}}\right\|_{W}+\left\|\theta_{z}-\theta_{z^{\prime}}\right\|_{Q} \\
\quad \leq c_{3}\left(\left\|z_{1}-z_{1}^{\prime}\right\|_{L^{2}\left(\Gamma_{3}\right)}+\left\|z_{2}-z_{2}^{\prime}\right\|_{L^{2}\left(\Gamma_{3}\right)}\right) .
\end{gathered}
$$

Hence the second part of Lemma 4 is proved. 
Remark 5. The second part of Lemma 4 implies that the function $z \mapsto\left(u_{z}, \varphi_{z}, \theta_{z}\right)$ where the triplet $\left(u_{z}, \varphi_{z}, \theta_{z}\right)$ is the solution of $\left(P V_{z}\right)$ is a continuous function from $L^{2}\left(\Gamma_{3}\right)^{2}$ to $V \times W \times Q$.

Lemma 6. If the triplet $\left(u_{z}, \varphi_{z}, \theta_{z}\right) \in V \times W \times Q$ is a solution of problem $\left(P V_{z}\right)$, then there exists a positive constant $\widetilde{c}_{1}$ such that

$$
\begin{aligned}
& \left\|u_{z}\right\|_{V} \\
& \quad \leq \widetilde{c}_{1}\left(\|f\|_{V}+\|\phi\|_{W}+\frac{1}{m_{\mathscr{K}}}\left(\|\mathcal{V}\|_{Q}+c_{2}\left\|z_{1}\right\|_{L^{2}\left(\Gamma_{3}\right)}\right)\right) .
\end{aligned}
$$

Proof. Taking $\eta=\theta_{z}$ in the variational equation (55), we get

$$
\left(\mathscr{K} \nabla \theta_{z}, \nabla \theta_{z}\right)_{H}=\left(\vartheta, \theta_{z}\right)_{\mathrm{Q}}-\chi_{1}\left(z, \theta_{z}\right) .
$$

Using (43), (30), and the ellipticity of the operator $\mathscr{K}$, we find

$$
\|\theta\|_{\mathrm{Q}} \leq \frac{1}{m_{\mathscr{K}}}\left(\|\vartheta\|_{\mathrm{Q}}+c_{2}\left\|z_{1}\right\|_{L^{2}\left(\Gamma_{3}\right)}\right) .
$$

Moreover, if we take $v=0 \in V$ in (53) and $\xi=\varphi_{z} \in W$ in (54), we have

$$
\begin{aligned}
& \left(\mathfrak{F}_{\varepsilon}\left(u_{z}\right), \varepsilon\left(u_{z}\right)\right)_{\mathscr{H}}+\left(\mathscr{E}^{*} \nabla \varphi_{z}, \varepsilon\left(u_{z}\right)\right)_{\mathscr{H}} \\
& \quad-\left(\mathscr{M} \theta_{z}, \varepsilon\left(u_{z}\right)\right)_{\mathscr{H}}+j_{n}\left(u_{z}, u_{z}\right) \\
& \quad+\chi_{2}\left(z, u_{z}\right) \leq\left(f, u_{z}\right)_{V}, \\
& \left(\beta \nabla \varphi_{z}, \nabla \varphi_{z}\right)_{H}-\left(\mathscr{E} \varepsilon\left(u_{z}\right), \nabla \varphi_{z}\right)_{H} \\
& \quad-\left(\mathscr{P} \theta_{z}, \nabla \varphi_{z}\right)_{H}=\left(\phi, \varphi_{z}\right)_{W} .
\end{aligned}
$$

Keeping in mind (6), the ellipticity of $\mathfrak{F}$ and $\beta$, the positivity of $j_{n}\left(u_{z}, u_{z}\right)$ and $\chi_{2}\left(z, u_{z}\right)$, and the properties of $\mathscr{M}$ and $\mathscr{P}$, we deduce that there exists a constant $\widetilde{c}_{1}>0$ such that

$$
\left\|u_{z}\right\|_{V}+\left\|\varphi_{z}\right\|_{W} \leq \widetilde{c}_{1}\left(\|f\|_{V}+\|\phi\|_{W}+\left\|\theta_{z}\right\|_{Q}\right) .
$$

We combine the two inequalities (91) and (93) to obtain

$$
\begin{aligned}
& \left\|u_{z}\right\|_{V} \\
& \quad \leq \widetilde{c}_{1}\left(\|f\|_{V}+\|\phi\|_{W}+\frac{1}{m_{\mathscr{K}}}\left(\|\vartheta\|_{Q}+c_{2}\left\|z_{1}\right\|_{L^{2}\left(\Gamma_{3}\right)}\right)\right),
\end{aligned}
$$

which finishes the proof.

Remark 7. Using the same argument as in the proof of in Lemma 6, we have that if the triplet $(u, \varphi, \theta) \in V \times W \times Q$ is a solution of problem $(P V)$, then

$$
\begin{aligned}
\|u\|_{V} & \leq \widetilde{c}_{1}\left(\|f\|_{V}+\|\phi\|_{W}\right. \\
& \left.+\frac{1}{m_{\mathscr{K}}}\left(\|\vartheta\|_{Q}+c_{2} M_{k} L \operatorname{meas}\left(\Gamma_{3}\right)^{1 / 2}\right)\right)=G,
\end{aligned}
$$

where $\widetilde{c}_{1}$ is the same constant as in (94).
In this step, we consider the operator $\Lambda: L^{2}\left(\Gamma_{3}\right)^{2} \rightarrow$ $L^{2}\left(\Gamma_{3}\right)^{2}$ defined by

$$
\Lambda z=\left(c_{n}\left(u_{z v}-g\right)_{+}^{m_{n}}, k_{c}\left(u_{z v}-g\right) \phi_{L}\left(\theta_{z}-\theta_{F}\right)\right) .
$$

Now, we shall prove that the operator $\Lambda$ has a unique fixed point $z^{*} \in L^{2}\left(\Gamma_{3}\right)^{2}$. For that, we need to introduce the two closed convex subsets of $L^{2}\left(\Gamma_{3}\right)$

$$
\begin{aligned}
& \mathscr{K}_{1}=\left\{z_{1} \geq 0 \in L^{2}\left(\Gamma_{3}\right),\left\|z_{1}\right\|_{L^{2}\left(\Gamma_{3}\right)} \leq k_{1}\right\}, \\
& \mathscr{K}_{2}=\left\{z_{2} \in L^{2}\left(\Gamma_{3}\right),\left\|z_{2}\right\|_{L^{2}\left(\Gamma_{3}\right)} \leq k_{2}\right\},
\end{aligned}
$$

where $k_{1}$ and $k_{2}$ will be defined below.

Lemma 8. For a specified values of $k_{1}$ and $k_{2}$, the operator $\Lambda$ has at least one fixed point.

Proof. Let $z=\left(z_{1}, z_{2}\right) \in \mathscr{K}_{1} \times \mathscr{K}_{2}$. We have $\left\|z_{1}\right\|_{L^{2}\left(\Gamma_{3}\right)} \leq k_{1}$ and $\left\|z_{2}\right\|_{L^{2}\left(\Gamma_{3}\right)} \leq k_{2}$ and then

$$
\|z\|_{L^{2}\left(\Gamma_{3}\right)^{2}} \leq k_{1}+k_{2} .
$$

On another hand, it follows from the definition of $\Lambda$ that

$$
\begin{aligned}
\|\Lambda z\|_{L^{2}\left(\Gamma_{3}\right)^{2}} \leq & \left\|c_{n}\left(u_{z v}-g\right)_{+}^{m_{n}}\right\|_{L^{2}\left(\Gamma_{3}\right)} \\
& +\left\|k_{c}\left(u_{z \nu}-g\right) \phi_{L}\left(\theta_{z}-\theta_{F}\right)\right\|_{L^{2}\left(\Gamma_{3}\right)} \\
\leq & \left\|c_{n}\right\|_{L^{\infty}\left(\Gamma_{3}\right)}\left\|u_{z v}-g\right\|_{L^{2 m_{n}\left(\Gamma_{3}\right)}}^{m_{n}} \\
& \quad+\left\|k_{c}\left(u_{z \nu}-g\right) \phi_{L}\left(\theta_{z}-\theta_{F}\right)\right\|_{L^{2}\left(\Gamma_{3}\right)} \\
\leq & \left\|c_{n}\right\|_{L^{\infty}\left(\Gamma_{3}\right)}\left(\left\|u_{z v}\right\|_{L^{2 m_{n}\left(\Gamma_{3}\right)}}+\|g\|_{L^{2 m_{n}\left(\Gamma_{3}\right)}}\right)^{m_{n}} \\
& +\left\|k_{c}\left(u_{z \nu}-g\right) \phi_{L}\left(\theta_{z}-\theta_{F}\right)\right\|_{L^{2}\left(\Gamma_{3}\right)} .
\end{aligned}
$$

Using $\left(h_{3}\right),\left(h_{8}\right)$, and Theorem 3, we deduce that there exists a constant $\tilde{c}>0$ such that

$$
\begin{gathered}
\|\Lambda z\|_{L^{2}\left(\Gamma_{3}\right)^{2}} \leq \tilde{c}\left\|c_{n}\right\|_{L^{\infty}\left(\Gamma_{3}\right)}\left(\left\|u_{z}\right\|_{V}+\|g\|_{L^{2 m_{n}\left(\Gamma_{3}\right)}}\right)^{m_{n}} \\
+M_{k} L \text { meas }\left(\Gamma_{3}\right)^{1 / 2} .
\end{gathered}
$$

Since $\left\|z_{1}\right\|_{L^{2}\left(\Gamma_{3}\right)} \leq k_{1}$, it becomes from Lemma 6 that there exists a constant $\widetilde{c}_{1}>0$ such that

$$
\begin{aligned}
\left\|u_{z}\right\|_{V} & \leq \widetilde{c}_{1}\left(\|f\|_{V}+\|\phi\|_{W}+\frac{1}{m_{\mathscr{K}}}\left(\|\vartheta\|_{Q}+c_{2} k_{1}\right)\right) \\
& =\widetilde{G} .
\end{aligned}
$$

If we choose $k_{1}=M_{k} L$ meas $\left(\Gamma_{3}\right)^{1 / 2}$ and $k_{2}=\widetilde{c}\left\|c_{n}\right\|_{L^{\infty}\left(\Gamma_{3}\right)}(\widetilde{G}+$ $\left.\|g\|_{L^{2 m_{n}\left(\Gamma_{3}\right)}}\right)^{m_{n}}$, we get

$$
\|\Lambda z\|_{L^{2}\left(\Gamma_{3}\right)^{2}} \leq k_{1}+k_{2} .
$$

Hence $\Lambda$ is an operator of $\mathscr{K}_{1} \times \mathscr{K}_{2}$ into itself. Since $\mathscr{K}_{1} \times$ $\mathscr{K}_{2}$ is a nonempty, convex, and closed subset of the reflexive space $L^{2}\left(\Gamma_{3}\right) \times L^{2}\left(\Gamma_{3}\right)$, then $\mathscr{K}_{1} \times \mathscr{K}_{2}$ is weakly compact. Using the continuity of the functions $\phi_{L}$ and $k_{c}$ and Remark 5 , we deduce that $\Lambda$ is a continuous operator. Hence, by Schauder's fixed point theorem the operator $\Lambda$ has a fixed point. 


\section{Proof of Theorem 1.}

Existence. Let $z^{*}$ be the fixed point of the operator $\Lambda$ obtained in Lemma 8 . We denote by $\left(u^{*}, \varphi^{*}, \theta^{*}\right)$ the solution of the problem $\left(P V_{z}\right)$ for $z=z^{*}$. It follows from the definition of $\Lambda$ and $\left(P V_{z}\right)$ that $\left(u^{*}, \varphi^{*}, \theta^{*}\right)$ is a solution of $(P V)$ which concludes the proof of the existence part.

Uniqueness. Here, we will show that there exists a positive constant $L^{*}$ such that $(P V)$ has a unique solution if $m_{T}+$ $\|\mathscr{P}\|+\|\mathscr{M}\|+L_{k} L+M_{k}<L^{*}$. We consider $\left(u_{1}, \varphi_{1}, \theta_{1}\right)$ and $\left(u_{2}, \varphi_{2}, \theta_{2}\right)$ two solutions of $(P V)$. From (46), we have

$$
\begin{aligned}
&(\mathfrak{F} \varepsilon\left.\left(u_{1}\right), \varepsilon(v)-\varepsilon\left(u_{1}\right)\right)_{\mathscr{H}} \\
& \quad+\left(\mathscr{E}^{*} \nabla \varphi_{1}, \varepsilon(v)-\varepsilon\left(u_{1}\right)\right)_{\mathscr{H}} \\
& \quad-\left(\mathscr{M} \theta_{1}, \varepsilon(v)-\varepsilon\left(u_{1}\right)\right)_{\mathscr{H}}+j_{n}\left(u_{1}, v-u_{1}\right) \\
& \quad+j_{T}\left(u_{1}, v\right)-j_{T}\left(u_{1}, u_{1}\right) \geq\left(f, v-u_{1}\right)_{V},
\end{aligned}
$$

$\forall v \in V$,

$$
\begin{aligned}
&(\mathfrak{F}\left.\varepsilon\left(u_{2}\right), \mathcal{\varepsilon}(v)-\varepsilon\left(u_{2}\right)\right)_{\mathscr{H}} \\
& \quad+\left(\mathscr{E}^{*} \nabla \varphi_{2}, \varepsilon(v)-\varepsilon\left(u_{2}\right)\right)_{\mathscr{H}} \\
& \quad-\left(\mathscr{M} \theta_{2}, \varepsilon(v)-\varepsilon\left(u_{2}\right)\right)_{\mathscr{H}}+j_{n}\left(u_{2}, v-u_{2}\right) \\
& \quad+j_{T}\left(u_{2}, v\right)-j_{T}\left(u_{2}, u_{2}\right) \geq\left(f, v-u_{2}\right)_{V},
\end{aligned}
$$

$\forall v \in V$.

After taking $v=u_{2}$ in the first inequality and $v=u_{1}$ in the second and adding the resulting inequalities, it follows from the positivity of $j_{n}\left(u_{1}-u_{2}, u_{1}-u_{2}\right)$ that

$$
\begin{aligned}
(\mathfrak{F} \varepsilon & \left.\left(u_{1}\right)-\mathfrak{F} \varepsilon\left(u_{2}\right), \varepsilon\left(u_{1}\right)-\varepsilon\left(u_{2}\right)\right)_{\mathscr{H}} \\
& +\left(\mathscr{E}^{*} \nabla \varphi_{1}-\mathscr{E}^{*} \nabla \varphi_{2}, \varepsilon\left(u_{1}\right)-\varepsilon\left(u_{2}\right)\right)_{\mathscr{H}} \\
& -\left(\mathscr{M} \theta_{1}-\mathscr{M} \theta_{2}, \varepsilon\left(u_{1}\right)-\varepsilon\left(u_{2}\right)\right)_{\mathscr{H}} \leq G_{1} \\
& =j_{T}\left(u_{1}, u_{2}\right)-j_{T}\left(u_{1}, u_{1}\right)+j_{T}\left(u_{2}, u_{1}\right) \\
& -j_{T}\left(u_{2}, u_{2}\right) .
\end{aligned}
$$

Plus the variational equation (47) leads to

$$
\begin{aligned}
& \left(\beta \nabla \varphi_{1}, \nabla \xi\right)_{H}-\left(\mathscr{E} \varepsilon\left(u_{1}\right), \nabla \xi\right)_{H}-\left(\mathscr{P} \theta_{1}, \nabla \xi\right)_{H} \\
& =(\phi, \xi)_{W}, \quad \forall \xi \in W, \\
& \left(\beta \nabla \varphi_{2}, \nabla \xi\right)_{H}-\left(\mathscr{E} \varepsilon\left(u_{2}\right), \nabla \xi\right)_{H}-\left(\mathscr{P} \theta_{2}, \nabla \xi\right)_{H} \\
& =(\phi, \xi)_{W}, \quad \forall \xi \in W .
\end{aligned}
$$

We take $\xi=\varphi_{1}-\varphi_{2}$ in the first equation and $\xi=\varphi_{2}-\varphi_{1}$ in the second to obtain

$$
\begin{aligned}
\left(\beta \nabla \varphi_{1}-\beta \nabla \varphi_{2} \nabla \varphi_{1}-\nabla \varphi_{2}\right)_{H} & \\
- & \left(\mathscr{E} \varepsilon\left(u_{1}\right)-\mathscr{E} \varepsilon\left(u_{2}\right), \nabla \varphi_{1}-\nabla \varphi_{2}\right)_{H} \\
& -\left(\mathscr{P} \theta_{1}-\mathscr{P} \theta_{2}, \nabla \varphi_{1}-\nabla \varphi_{2}\right)_{H}=0 .
\end{aligned}
$$

Hence, the addition of (104) and (106) implies that

$$
\begin{aligned}
(\mathfrak{F} \varepsilon & \left.\left(u_{1}\right)-\mathfrak{F} \varepsilon\left(u_{2}\right), \varepsilon\left(u_{1}\right)-\varepsilon\left(u_{2}\right)\right)_{\mathscr{H}} \\
& +\left(\beta \nabla \varphi_{1}-\beta \nabla \varphi_{2}, \nabla \varphi_{1}-\nabla \varphi_{2}\right)_{H} \\
& -\left(\mathscr{P} \theta_{1}-\mathscr{P} \theta_{2}, \nabla \varphi_{1}-\nabla \varphi_{2}\right)_{H} \\
& -\left(\mathscr{M} \theta_{1}-\mathscr{M} \theta_{2}, \varepsilon\left(u_{1}\right)-\varepsilon\left(u_{2}\right)\right)_{\mathscr{H}} \leq G_{1} .
\end{aligned}
$$

Moreover, we use the variational equation (48) to deduce

$$
\begin{array}{ll}
\left(\mathscr{K} \nabla \theta_{1}, \nabla \eta\right)_{H}+\chi\left(u_{1}, \theta_{1}, \eta\right)=(\vartheta, \eta)_{\mathrm{Q}}, & \forall \eta \in Q, \\
\left(\mathscr{K} \nabla \theta_{2}, \nabla \eta\right)_{H}+\chi\left(u_{2}, \theta_{2}, \eta\right)=(\vartheta, \eta)_{\mathrm{Q}}, & \forall \eta \in Q .
\end{array}
$$

We substitute $\eta$ by $\theta_{1}-\theta_{2}$ and we subtract the two induced equations to obtain

$$
\begin{aligned}
& \left(\mathscr{K} \nabla \theta_{1}-\mathscr{K} \nabla \theta_{2}, \nabla \theta_{1}-\nabla \theta_{2}\right)_{H}+\chi\left(u_{1}, \theta_{1}, \theta_{1}-\theta_{2}\right) \\
& \quad-\chi\left(u_{2}, \theta_{2}, \theta_{1}-\theta_{2}\right)=0 .
\end{aligned}
$$

Let us consider $\Delta_{1}=\chi\left(u_{1}, \theta_{1}, \theta_{1}-\theta_{2}\right)-\chi\left(u_{2}, \theta_{2}, \theta_{1}-\theta_{2}\right)$. Then, we have

$$
\begin{aligned}
\Delta_{1} & =\int_{\Gamma_{3}}\left[k_{c}\left(u_{1 v}-g\right) \phi_{L}\left(\theta_{1}-\theta_{F}\right)\right. \\
& \left.-k_{c}\left(u_{2 v}-g\right) \phi_{L}\left(\theta_{2}-\theta_{F}\right)\right]\left(\theta_{1}-\theta_{2}\right) d a \\
& \leq\left\|\Delta_{2}\right\|_{L^{2}\left(\Gamma_{3}\right)}\left\|\theta_{1}-\theta_{2}\right\|_{L^{2}\left(\Gamma_{3}\right)},
\end{aligned}
$$

where

$$
\begin{aligned}
\Delta_{2}= & k_{c}\left(u_{1 v}-g\right) \phi_{L}\left(\theta_{1}-\theta_{F}\right) \\
& -k_{c}\left(u_{2 v}-g\right) \phi_{L}\left(\theta_{2}-\theta_{F}\right) \\
= & k_{c}\left(u_{1 v}-g\right) \phi_{L}\left(\theta_{1}-\theta_{F}\right) \\
& -k_{c}\left(u_{2 v}-g\right) \phi_{L}\left(\theta_{1}-\theta_{F}\right) \\
& +k_{c}\left(u_{2 v}-g\right) \phi_{L}\left(\theta_{1}-\theta_{F}\right) \\
& -k_{c}\left(u_{2 v}-g\right) \phi_{L}\left(\theta_{2}-\theta_{F}\right) .
\end{aligned}
$$

Taking in mind (26), (30), and the assumptions $\left(\mathrm{h}_{3}\right)$ and $\left(\mathrm{h}_{4}\right)$, we get

$$
\left\|\Delta_{2}\right\|_{L^{2}\left(\Gamma_{3}\right)} \leq c_{0} L_{k} L\left\|u_{1}-u_{2}\right\|_{V}+c_{2} M_{k}\left\|\theta_{1}-\theta_{2}\right\|_{Q} .
$$

Hence, it comes from (109), (110), and (112) and the ellipticity of $\mathscr{K}$ that

$$
\begin{aligned}
m_{\mathscr{K}}\left\|\theta_{1}-\theta_{2}\right\|_{\mathrm{Q}}^{2} \leq & L_{k} L c_{0} c_{2}\left\|u_{1}-u_{2}\right\|_{V}\left\|\theta_{1}-\theta_{2}\right\|_{\mathrm{Q}} \\
& +M_{k} c_{2}^{2}\left\|\theta_{1}-\theta_{2}\right\|_{\mathrm{Q}}^{2} .
\end{aligned}
$$

Recalling $a b \leqslant(1 / 4) a^{2}+b^{2}$, forall $a, b \in \mathbb{R}$, we deduce

$$
\begin{aligned}
& m_{\mathscr{K}}\left\|\theta_{1}-\theta_{2}\right\|_{Q}^{2} \\
& \quad \leq\left(\frac{5}{4} L_{k} L c_{0} c_{2}+M_{k} c_{2}^{2}\right)\left(\left\|u_{1}-u_{2}\right\|_{V}^{2}+\left\|\theta_{1}-\theta_{2}\right\|_{Q}^{2}\right) .
\end{aligned}
$$


Furthermore, the definition of $j_{T}$ implies that

$$
\begin{aligned}
G_{1} & =j_{T}\left(u_{1}, u_{2}\right)-j_{T}\left(u_{1}, u_{1}\right)+j_{T}\left(u_{2}, u_{1}\right) \\
& -j_{T}\left(u_{2}, u_{2}\right)=\int_{\Gamma_{3}} c_{T}\left[\left(u_{1 v}-g\right)_{+}^{m_{T}}-\left(u_{2 v}-g\right)_{+}^{m_{T}}\right] \\
& \cdot\left(\left|u_{1 \tau}\right|-\left|u_{2 \tau}\right|\right) d a \leq\left\|c_{T}\right\|_{L^{\infty}\left(\Gamma_{3}\right)} \|\left(u_{1 v}-g\right)_{+}^{m_{T}} \\
& -\left(u_{2 v}-g\right)_{+}^{m_{T}}\left\|_{L^{4 / 3}\left(\Gamma_{3}\right)}\right\| u_{1}-u_{2} \|_{L^{4}\left(\Gamma_{3}\right)^{d}} .
\end{aligned}
$$

Using the following mathematical inequalities (see [23])

$$
\left(a^{m}-b^{m}\right) \leq m|a-b|\left(a^{m-1}-b^{m-1}\right)
$$

$(\forall a, b \geq 0, \forall m>1)$

$$
\left|(a)_{+}-(b)_{+}\right| \leq|a-b|, \quad(\forall a, b \in \mathbb{R}) .
$$

Then, we obtain

$$
\begin{aligned}
& \left\|\left(u_{1 v}-g\right)_{+}^{m_{T}}-\left(u_{2 v}-g\right)_{+}^{m_{T}}\right\|_{L^{4 / 3}\left(\Gamma_{3}\right)} \\
& =\left[\int_{\Gamma_{3}}\left|\left(u_{1 v}-g\right)_{+}^{m_{T}}-\left(u_{2 v}-g\right)_{+}^{m_{T}}\right|^{4 / 3} d a\right]^{3 / 4} \\
& \leq m_{T}\left\|u_{1 v}-u_{2 v}\right\|^{L^{4}\left(\Gamma_{3}\right)} \\
& \cdot\left\|\left(u_{1 v}-g\right)_{+}^{m_{T}-1}+\left(u_{2 v}-g\right)_{+}^{m_{T}-1}\right\|_{L^{2}\left(\Gamma_{3}\right)} \\
& \leq m_{T}\left\|u_{1}-u_{2}\right\|_{L^{4}\left(\Gamma_{3}\right)^{d}} \\
& \quad \cdot\left[\left\|\left(u_{1 v}-g\right)_{+}^{m_{T}-1}\right\|_{L^{2}\left(\Gamma_{3}\right)}+\left\|\left(u_{2 v}-g\right)_{+}^{m_{T}-1}\right\|_{L^{2}\left(\Gamma_{3}\right)}\right] .
\end{aligned}
$$

Keeping in mind Theorem 3 and Remark 7, we obtain

$$
G_{1} \leq m_{T} R\left\|u_{1}-u_{2}\right\|_{V}^{2}
$$

where $R=2 N_{4}^{2}\left\|\mathcal{C}_{T}\right\|_{L^{\infty}\left(\Gamma_{3}\right)}\left[N_{2\left(m_{T}-1\right)}^{m_{T}-1} G^{m_{T}-1}+\left\||g|^{m_{T}-1}\right\|_{L^{2}\left(\Gamma_{3}\right)}\right]$.

Using (107), (114), and (118), after some algebra it follows that there exists $\widetilde{C}>0$ such that

$$
\begin{gathered}
\left\|u_{1}-u_{2}\right\|_{V}^{2}+\left\|\varphi_{1}-\varphi_{2}\right\|_{W}^{2}+\left\|\theta_{1}-\theta_{2}\right\|_{Q}^{2} \\
\leq \widetilde{C}\left(m_{T}+\|\mathscr{P}\|+\|\mathscr{M}\|+L_{k} L+M_{k}\right) \\
\cdot\left[\left\|u_{1}-u_{2}\right\|_{V}^{2}+\left\|\varphi_{1}-\varphi_{2}\right\|_{W}^{2}+\left\|\theta_{1}-\theta_{2}\right\|_{Q}^{2}\right],
\end{gathered}
$$

where

$$
\widetilde{C}=\frac{\max \left\{1, R, c_{0} c_{2}, c_{2}^{2}\right\}}{\min \left\{m_{\mathscr{F}}, m_{\beta}, m_{\mathscr{K}}\right\}} .
$$

Choose $L^{*}=1 / \widetilde{C}$. Then, if $m_{T}+\|\mathscr{P}\|+\|\mathscr{M}\|+L_{k} L+M_{k}<$ $L^{*}$ holds, we will conclude that $u_{1}=u_{2}, \varphi_{1}=\varphi_{2}$, and $\theta_{1}=\theta_{2}$ which leads to the uniqueness part of Theorem 1 .

\section{Discrete Approximation}

This section deals with the discrete approximation of the problem $(P V)$. We assume that the conditions $\left(\mathrm{h}_{1}\right)-\left(\mathrm{h}_{8}\right)$ hold, then the problem $(P V)$ has a unique solution $(u, \varphi, \theta) \in V \times$ $W \times Q$. Let $\mathscr{T}_{h}=\left(T_{r}\right)_{r \in \mathscr{G}_{h}}$ be a family of regular triangulations of the polygonal domain $\Omega$ such that

$$
\begin{aligned}
\bigcup_{r} \bar{T}_{r} & =\bar{\Omega}, \\
T_{r} \cap T_{r^{\prime}} & =\emptyset,
\end{aligned}
$$

$$
\forall r \neq r^{\prime} \in \mathscr{G}_{h} .
$$

Here and below $h>0$ is a discretization parameter. We define the following finite dimensional subspaces $V^{h}, W^{h}$, and $Q^{h}$ which approximate, respectively, the spaces $V, W$, and $Q$ by

$$
\begin{aligned}
V^{h} & =\left\{v^{h} \in C(\bar{\Omega})^{d} ;\left(\forall r \in \mathscr{G}_{h}\right), v_{\mid T_{r}}^{h}\right. \\
& \left.\in\left(\mathbb{P}_{1}\left(T_{r}\right)\right)^{d}, v^{h}=0 \text { on } \Gamma_{1}\right\}, \\
W^{h} & =\left\{\xi^{h} \in C(\bar{\Omega}) ;\left(\forall r \in \mathscr{G}_{h}\right), \xi_{\mid T_{r}}^{h} \in \mathbb{P}_{1}\left(T_{r}\right), \xi^{h}\right. \\
& \left.=0 \text { on } \Gamma_{a}\right\}, \\
Q^{h} & =\left\{\eta^{h} \in C(\bar{\Omega}) ;\left(\forall r \in \mathscr{G}_{h}\right), \eta_{\mid T_{r}}^{h} \in \mathbb{P}_{1}\left(T_{r}\right), \eta^{h}\right. \\
& \left.=0 \text { on } \Gamma_{1}\right\},
\end{aligned}
$$

where $\mathbb{P}_{1}\left(T_{r}\right)$ denotes the space of polynomials of a degree lower or equal to one on $T_{r}$. Then, the discrete approximation of the problem $(P V)$ is as follows.

Problem $\left(P V^{h}\right)$. Find the displacement $u^{h} \in V^{h}$, the electric potential $\varphi^{h} \in W^{h}$, and the temperature $\theta^{h} \in Q^{h}$ such that

$$
\begin{aligned}
& \left(\mathfrak{F}_{\varepsilon}\left(u^{h}\right), \varepsilon\left(v^{h}\right)-\varepsilon\left(u^{h}\right)\right)_{\mathscr{H}} \\
& +\left(\mathscr{E}^{*} \nabla \varphi^{h}, \varepsilon\left(v^{h}\right)-\varepsilon\left(u^{h}\right)\right)_{\mathscr{H}} \\
& \quad-\left(\mathscr{M} \theta, \varepsilon\left(v^{h}\right)-\varepsilon\left(u^{h}\right)\right)_{\mathscr{H}}+j_{n}\left(u^{h}, v^{h}-u^{h}\right) \\
& +j_{T}\left(u^{h}, v^{h}\right)-j_{T}\left(u^{h}, u^{h}\right) \geq\left(f, v^{h}-u^{h}\right)_{V}, \\
& \left(\beta \nabla \varphi^{h}, \nabla \xi^{h}\right)_{H}-\left(\mathscr{E} \varepsilon\left(u^{h}\right), \nabla \xi^{h}\right)_{H} \quad \forall v^{h} \in V^{h}, \\
& -\left(\mathscr{P} \theta^{h}, \nabla \xi^{h}\right)_{H}=\left(\phi, \xi^{h}\right)_{W}, \quad \forall \xi^{h} \in W^{h}, \\
& \left(\mathscr{K} \nabla \theta^{h}, \nabla \eta^{h}\right)_{H}+\chi\left(u^{h}, \theta^{h}, \eta^{h}\right)=\left(\vartheta, \eta^{h}\right)_{Q},
\end{aligned}
$$

Under the assumptions of Theorem 1 and with the same arguments, we can prove that the discrete problem $\left(P V^{h}\right)$ 
has a unique solution $\left(u^{h}, \varphi^{h}, \theta^{h}\right)$ in $V^{h} \times W^{h} \times Q^{h}$. Now, we proceed to derive some error estimates for the discrete solution. In the sequel, $c$ denotes positive constants which are independent of the discretization parameter $h$.

Theorem 9. Assume the conditions of Theorem 1 hold. One has the following error estimate:

$$
\begin{gathered}
\left\|u-u^{h}\right\|_{V}^{2}+\left\|\varphi-\varphi^{h}\right\|_{W}^{2}+\left\|\theta-\theta^{h}\right\|_{Q}^{2} \leq c\left(\left\|u-v^{h}\right\|_{V}^{2}\right. \\
\left.+\left\|u-v^{h}\right\|_{V}+\left\|\varphi-\xi^{h}\right\|_{W}^{2}+\left\|\theta-\eta^{h}\right\|_{Q}^{2}\right) .
\end{gathered}
$$

Proof. Taking $\xi=\xi^{h} \in W^{h} \subset W$ in the second equation of the problem $(P V)$ and subtracting the obtained equation to the second equation of the problem $\left(P V^{h}\right)$, we obtain

$$
\begin{gathered}
\left(\beta \nabla \varphi-\beta \nabla \varphi^{h}, \nabla \xi^{h}\right)_{H}-\left(\mathscr{E} \varepsilon(u)-\mathscr{E} \varepsilon\left(u^{h}\right), \nabla \xi^{h}\right)_{H} \\
-\left(\mathscr{P} \theta-\mathscr{P} \theta^{h}, \nabla \xi^{h}\right)_{H}=0 .
\end{gathered}
$$

We substitute $\xi^{h}$ by $\xi^{h}-\varphi^{h}=\left(\xi^{h}-\varphi\right)+\left(\varphi-\varphi^{h}\right)$ to find after simplifications

$$
\begin{aligned}
\left(\mathscr{E} \varepsilon(u)-\mathscr{E} \varepsilon\left(u^{h}\right), \nabla \varphi-\nabla \varphi^{h}\right)_{H} \\
=\left(\beta \nabla \varphi-\beta \nabla \varphi^{h}, \nabla \varphi-\nabla \varphi^{h}\right)_{H} \\
\quad+\left(\beta \nabla \varphi-\beta \nabla \varphi^{h}, \nabla \xi^{h}-\nabla \varphi\right)_{H} \\
\quad-\left(\mathscr{P} \theta-\mathscr{P} \theta^{h}, \nabla \varphi-\nabla \varphi^{h}\right)_{H} \\
\quad-\left(\mathscr{P} \theta-\mathscr{P} \theta^{h}, \nabla \xi^{h}-\nabla \varphi\right)_{H} \\
-\left(\mathscr{E} \varepsilon(u)-\mathscr{E} \varepsilon\left(u^{h}\right), \nabla \xi^{h}-\nabla \varphi\right)_{H} .
\end{aligned}
$$

Take $v=u^{h} \in V^{h} \subset V$ in the first inequality of the problem $(P V)$ to deduce

$$
\begin{aligned}
\left(\mathfrak{F}^{\varepsilon}\right. & \left.(u), \varepsilon\left(u^{h}\right)-\varepsilon(u)\right)_{\mathscr{H}} \\
& +\left(\mathscr{E}^{*} \nabla \varphi, \varepsilon\left(u^{h}\right)-\varepsilon(u)\right)_{\mathscr{H}} \\
& -\left(\mathscr{M} \theta, \varepsilon\left(u^{h}\right)-\varepsilon(u)\right)_{\mathscr{C}}+j_{n}\left(u, u^{h}-u\right) \\
& +j_{T}\left(u, u^{h}\right)-j_{T}(u, u) \geq\left(f, u^{h}-u\right)_{V} .
\end{aligned}
$$

By using $v^{h}-u^{h}=\left(u-u^{h}\right)-\left(u-v^{h}\right)$, it follows from the first inequality of $\left(P V^{h}\right)$ that

$$
\begin{aligned}
\left(\mathfrak{F}_{\varepsilon}\right. & \left.\left(u^{h}\right), \varepsilon(u)-\varepsilon\left(u^{h}\right)\right)_{\mathscr{H}} \\
& +\left(\mathscr{E}^{*} \nabla \varphi^{h}, \varepsilon(u)-\varepsilon\left(u^{h}\right)\right)_{H} \\
& -\left(\mathscr{M} \theta^{h}, \varepsilon(u)-\varepsilon\left(u^{h}\right)\right)_{\mathscr{H}}+j_{n}\left(u^{h}, u-u^{h}\right) \\
& -j_{n}\left(u^{h}, u-v^{h}\right)+j_{T}\left(u^{h}, v^{h}\right)-j_{T}\left(u^{h}, u^{h}\right) \\
\geq & \left(\mathfrak{F}_{\varepsilon}\left(u^{h}\right), \varepsilon(u)-\varepsilon\left(v^{h}\right)\right)_{\mathscr{H}}
\end{aligned}
$$

$$
\begin{array}{r}
-\left(\mathscr{M} \theta^{h}, \varepsilon(u)-\varepsilon\left(v^{h}\right)\right)_{\mathscr{H}} \\
+\left(\mathscr{E}^{*} \nabla \varphi^{h}, \varepsilon(u)-\varepsilon\left(v^{h}\right)\right)_{H}+\left(f, v^{h}-u^{h}\right)_{V}, \\
\forall v^{h} \in V^{h} .
\end{array}
$$

From Lemma 2, we know that $j_{n}\left(u^{h}, u-u^{h}\right)+j_{n}\left(u, u^{h}-u\right) \geq$ 0 . Then, keeping in mind t (128) and (6), the addition of (129) and (130) gives

$$
\begin{aligned}
(\mathfrak{F} \varepsilon & \left.(u)-\mathfrak{F} \varepsilon\left(u^{h}\right), \varepsilon(u)-\varepsilon\left(u^{h}\right)\right)_{\mathscr{C}} \\
& +\left(\beta \nabla \varphi-\beta \nabla \varphi^{h}, \nabla \varphi-\nabla \varphi^{h}\right)_{H} \\
\leq & \left(\mathscr{P} \theta-\mathscr{P} \theta^{h}, \nabla \varphi-\nabla \varphi^{h}\right)_{H} \\
& +\left(\mathscr{M} \theta-\mathscr{M} \theta^{h}, \varepsilon(u)-\varepsilon\left(u^{h}\right)\right)_{\mathscr{H}}+I_{1}+I_{2},
\end{aligned}
$$

where

$$
\begin{aligned}
I_{1}= & \left(\mathfrak{F} \varepsilon\left(u^{h}\right), \varepsilon\left(v^{h}\right)-\varepsilon(u)\right)_{\mathscr{C}} \\
& +\left(\mathscr{E} \varepsilon(u)-\mathscr{E} \varepsilon\left(u^{h}\right), \nabla \xi^{h}-\nabla \varphi\right)_{H} \\
& -\left(\beta \nabla \varphi-\beta \nabla \varphi^{h}, \nabla \xi^{h}-\nabla \varphi\right)_{H} \\
& +\left(\mathscr{P} \theta-\mathscr{P} \theta^{h}, \nabla \xi^{h}-\nabla \varphi\right)_{H} \\
& -j_{n}\left(u^{h}, u-v^{h}\right)+\left(\mathscr{u} \theta^{h}, \varepsilon(u)-\varepsilon\left(v^{h}\right)\right)_{\mathscr{H}} \\
& -\left(\mathscr{E}^{*} \nabla \varphi^{h}, \varepsilon(u)-\varepsilon\left(v^{h}\right)\right)_{H}-\left(f, v^{h}-u\right)_{V} . \\
I_{2}= & j_{T}\left(u^{h}, v^{h}\right)-j_{T}\left(u^{h}, u^{h}\right)+j_{T}\left(u, u^{h}\right) \\
& -j_{T}(u, u) .
\end{aligned}
$$

Next, we rewrite (48) for $\eta=\eta^{h} \in Q^{h} \subset Q$ and we subtract it from (125) to obtain

$$
\begin{aligned}
& \left(\mathscr{K} \nabla \theta^{h}-\mathscr{K} \nabla \theta, \nabla \eta^{h}\right)_{H} \\
& \quad+\int_{\Gamma_{3}}\left[k\left(u_{v}^{h}-g\right) \phi_{L}\left(\theta^{h}-\theta_{F}\right)\right. \\
& \left.-k\left(u_{v}-g\right) \phi_{L}\left(\theta-\theta_{F}\right)\right] \eta^{h} d a=0 .
\end{aligned}
$$

We replace $\eta^{h}$ by $\eta^{h}-\theta^{h}=\left(\eta^{h}-\theta\right)+\left(\theta-\theta^{h}\right)$. Hence, we have

$$
\begin{aligned}
& \left(\mathscr{K} \nabla \theta^{h}-\mathscr{K} \nabla \theta, \nabla \theta-\nabla \theta^{h}\right)_{H}=\left(\mathscr{K} \nabla \theta^{h}-\mathscr{K} \nabla \theta, \nabla \theta\right. \\
& \left.-\nabla \eta^{h}\right)_{H}+\int_{\Gamma_{3}}\left[k\left(u_{\nu}^{h}-g\right) \phi_{L}\left(\theta^{h}-\theta_{F}\right)\right. \\
& \left.-k\left(u_{v}-g\right) \phi_{L}\left(\theta-\theta_{F}\right)\right]\left(\theta-\eta^{h}\right) d a \\
& -\int_{\Gamma_{3}}\left[k\left(u_{v}^{h}-g\right) \phi_{L}\left(\theta^{h}-\theta_{F}\right)\right. \\
& \left.-k\left(u_{v}-g\right) \phi_{L}\left(\theta-\theta_{F}\right)\right]\left(\theta-\theta^{h}\right) d a .
\end{aligned}
$$


By adding the two results (134) and (131), we deduce the following inequality:

$$
\begin{aligned}
\left(\mathfrak{F} \varepsilon(u)-\mathfrak{F} \varepsilon\left(u^{h}\right), \varepsilon(u)-\varepsilon\left(u^{h}\right)\right)_{\mathscr{H}} \\
\quad+\left(\beta \nabla \varphi-\beta \nabla \varphi^{h}, \nabla \varphi-\nabla \varphi^{h}\right)_{H} \\
\quad+\left(\mathscr{K} \nabla \theta-\mathscr{K} \nabla \theta^{h}, \nabla \theta-\nabla \theta^{h}\right)_{H} \\
\leq I_{1}+I_{2}+I_{3}+I_{4},
\end{aligned}
$$

where $I_{3}$ and $I_{4}$ are given by

$$
\begin{aligned}
I_{3} & =\left(\mathscr{K} \nabla \theta-\mathscr{K} \nabla \theta^{h}, \nabla \theta-\nabla \eta^{h}\right)_{H} \\
& +\int_{\Gamma_{3}}\left[k\left(u_{v}^{h}-g\right) \phi_{L}\left(\theta^{h}-\theta_{F}\right)\right. \\
& \left.-k\left(u_{v}-g\right) \phi_{L}\left(\theta-\theta_{F}\right)\right]\left(\eta^{h}-\theta\right) d a, \\
I_{4} & =\left(\mathscr{P} \theta-\mathscr{P} \theta^{h}, \nabla \varphi-\nabla \varphi^{h}\right)_{L^{2}(\Omega)^{d}}+\left(\mathscr{M} \theta-\mathscr{M} \theta^{h},\right. \\
& \left.\varepsilon(u)-\varepsilon\left(u^{h}\right)\right)_{\mathscr{H}}-\int_{\Gamma_{3}}\left[k\left(u_{v}^{h}-g\right) \phi_{L}\left(\theta^{h}-\theta_{F}\right)\right. \\
& \left.-k\left(u_{v}-g\right) \phi_{L}\left(\theta-\theta_{F}\right)\right]\left(\theta^{h}-\theta\right) d a .
\end{aligned}
$$

Now, we begin to estimate the quantities $I_{1}, I_{2}, I_{3}$, and $I_{4}$. We have

$$
\begin{aligned}
I_{1} \leq & \|\mathfrak{F}\|\left\|u-u^{h}\right\|_{V}\left\|u-v^{h}\right\|_{V}+\|\mathfrak{F}\|\|u\|_{V}\left\|u-v^{h}\right\|_{V} \\
& +\|\mathscr{E}\|\left\|u-u^{h}\right\|_{V}\left\|\xi^{h}-\varphi\right\|_{W} \\
& +\|\beta\|\left\|\varphi-\varphi^{h}\right\|_{W}\left\|\xi^{h}-\varphi\right\|_{W} \\
& +\|\mathscr{P}\|\left\|\theta-\theta^{h}\right\|_{Q}\left\|\xi^{h}-\varphi\right\|_{W}+\|f\|_{V}\left\|v^{h}-u\right\|_{V} \\
& +\|\mathscr{M}\|\left\|\theta-\theta^{h}\right\|_{Q}\left\|u-v^{h}\right\|_{V} \\
& +\|\mathscr{M}\|\|\theta\|_{Q}\left\|u-v^{h}\right\|_{V}+\left\|\mathscr{E}^{*}\right\|\|\varphi\|_{W}\left\|u-v^{h}\right\|_{V} \\
& +\left\|c_{n}\left(u_{v}^{h}-g\right)_{+}^{m_{n}}\right\|_{L^{4 / 3}\left(\Gamma_{3}\right)}\left\|v^{h}-u\right\|_{L^{4}\left(\Gamma_{3}\right)^{d}} \\
& +\left\|\mathscr{E}^{*}\right\|\left\|\varphi-\varphi^{h}\right\|_{W}\left\|u-v^{h}\right\|_{V} \cdot
\end{aligned}
$$

We recall that there exists a positive constant $c$ independent of $h$ such that

$$
\begin{gathered}
\left\|c_{n}\left(u_{v}^{h}-g\right)_{+}^{m_{n}}\right\|_{L^{4 / 3}\left(\Gamma_{3}\right)}\left\|v^{h}-u\right\|_{L^{4}\left(\Gamma_{3}\right)^{d}} \leq c\left\|c_{n}\right\|_{L^{\infty}\left(\Gamma_{3}\right)} \\
\cdot\left\|u_{v}^{h}-g\right\|_{L^{4 m_{n} / 3}\left(\Gamma_{3}\right)}^{m_{n}}\left\|v^{h}-u\right\|_{V} \leq c\left\|c_{n}\right\|_{L^{\infty}\left(\Gamma_{3}\right)} \\
\cdot\left(N_{4 m_{n} / 3}^{m_{n}}\left\|u^{h}\right\|_{V}^{m_{n}}+\|g\|_{L^{4 m_{n} / 3}\left(\Gamma_{3}\right)}^{m_{n}}\right)\left\|v^{h}-u\right\|_{V}
\end{gathered}
$$

and similar to Remark 7, we can find that $\left\|u^{h}\right\|_{V} \leq G$. Thus

$$
\begin{gathered}
\left\|c_{n}\left(u_{v}^{h}-g\right)_{+}^{m_{n}}\right\|_{L^{4 / 3}\left(\Gamma_{3}\right)}\left\|v^{h}-u\right\|_{L^{4}\left(\Gamma_{3}\right)^{d}} \leq c\left\|c_{n}\right\|_{L^{\infty}\left(\Gamma_{3}\right)} \\
\cdot\left(N_{4 m_{n} / 3}^{m_{n}} G^{m_{n}}+\|g\|_{L^{4 m_{n} / 3}\left(\Gamma_{3}\right)}^{m_{n}}\right)\left\|v^{h}-u\right\|_{V} .
\end{gathered}
$$

Finally, remembering (95), we find that there exists a constant $\alpha_{1}>0$ such that

$$
\begin{aligned}
I_{1} & \leq \alpha_{1}\left\{\left\|v^{h}-u\right\|_{V}+\left\|u^{h}-u\right\|_{V}\left\|v^{h}-u\right\|_{V}\right. \\
& +\left\|u^{h}-u\right\|_{V}\left\|\xi^{h}-\varphi\right\|_{W}+\left\|v^{h}-u\right\|_{V}\left\|\theta^{h}-\theta\right\|_{Q} \\
& +\left\|\varphi-\varphi^{h}\right\|_{W}\left\|\xi^{h}-\varphi\right\|_{W}+\left\|\theta^{h}-\theta\right\|_{Q}\left\|\xi^{h}-\varphi\right\|_{W} \\
& \left.+\left\|v^{h}-u\right\|_{V}\left\|\varphi^{h}-\varphi\right\|_{W}\right\} .
\end{aligned}
$$

Using the same process as in the proof of (118), we can deduce

$$
\begin{aligned}
& I_{2}=j_{T}\left(u^{h}, v^{h}\right)-j_{T}\left(u^{h}, u^{h}\right)+j_{T}\left(u, u^{h}\right)-j_{T}(u, u) \\
& =j_{T}\left(u, u^{h}\right)-j_{T}\left(u^{h}, u^{h}\right)+j_{T}\left(u^{h}, u\right)-j_{T}(u, u) \\
& +j_{T}\left(u^{h}, v^{h}\right)-j_{T}\left(u, v^{h}\right)+j_{T}(u, u)-j_{T}\left(u^{h}, u\right) \\
& +j_{T}\left(u, v^{h}\right)-j_{T}(u, u) \\
& =\int_{\Gamma_{3}} c_{T}\left[\left(u_{v}-g\right)_{+}^{m_{T}}-\left(u_{\nu}^{h}-g\right)_{+}^{m_{T}}\right] \\
& \cdot\left[\left|u_{\tau}^{h}\right|-\left|u_{\tau}\right|\right] d a \\
& +\int_{\Gamma_{3}} c_{T}\left[\left(u_{v}-g\right)_{+}^{m_{T}}-\left(u_{v}^{h}-g\right)_{+}^{m_{T}}\right] \\
& \cdot\left[\left|v_{\tau}^{h}\right|-\left|u_{\tau}\right|\right] d a+\int_{\Gamma_{3}} c_{T}\left(u_{v}-g\right)_{+}^{m_{T}} \\
& \cdot\left[\left|v_{\tau}^{h}\right|-\left|u_{\tau}\right|\right] d a \leq m_{T} R\left\|u^{h}-u\right\|_{V}^{2}+m_{T} R \| u^{h} \\
& -u\left\|_{V}\right\| v^{h}-u\left\|_{V}+\right\| c_{T} \|_{L^{\infty}\left(\Gamma_{3}\right)}\left(N_{2 m_{T}}^{m_{T}} G^{m_{T}}\right. \\
& \left.+\|g\|_{L^{2 m_{T}\left(\Gamma_{3}\right)}}^{m_{T}}\right)\left\|v^{h}-u\right\|_{L^{2}\left(\Gamma_{3}\right)^{d}} \leq m_{T} R\left\|u^{h}-u\right\|_{V}^{2} \\
& +\alpha_{2}\left(\left\|u^{h}-u\right\|_{V}\left\|v^{h}-u\right\|_{V}+\left\|v^{h}-u\right\|_{V}\right),
\end{aligned}
$$

where $\alpha_{2}$ is a positive constant. Moreover, we have

$$
\begin{aligned}
I_{3} & \leq\|\mathscr{K}\|\left\|\theta-\theta^{h}\right\|_{\mathrm{Q}}\left\|\theta-\eta^{h}\right\|_{\mathrm{Q}}+M_{k} c_{2}\left\|\theta^{h}-\theta\right\|_{\mathrm{Q}} \| \theta \\
& -\eta^{h}\left\|_{L^{2}\left(\Gamma_{3}\right)}+L L_{k} c_{0}\right\| u^{h}-u\left\|_{V}\right\| \theta-\eta^{h} \|_{L^{2}\left(\Gamma_{3}\right)} \\
& \leq \alpha_{3}\left(\left\|\theta-\theta^{h}\right\|_{Q}\left\|\theta-\eta^{h}\right\|_{Q}+\left\|\theta^{h}-\theta\right\|_{Q}\left\|\theta-\eta^{h}\right\|_{Q}\right. \\
& \left.+\left\|u^{h}-u\right\|_{V}\left\|\theta-\eta^{h}\right\|_{Q}\right),
\end{aligned}
$$

where $\alpha_{3}$ is a positive constant. Finally, we have

$$
\begin{aligned}
I_{4} \leq & \|\mathscr{P}\|\left\|\theta-\theta^{h}\right\|_{Q}\left\|\varphi-\varphi^{h}\right\|_{W} \\
& +\|\mathscr{M}\|\left\|\theta-\theta^{h}\right\|_{Q}\left\|u-u^{h}\right\|_{V}+M_{k} c_{2}^{2}\left\|\theta-\theta^{h}\right\|_{Q}^{2} \\
& +L L_{k} c_{1} c_{2}\left\|\theta-\theta^{h}\right\|_{Q}\left\|u-u^{h}\right\|_{V}
\end{aligned}
$$




$$
\begin{aligned}
\leq & \|\mathscr{P}\|\left(\left\|\theta-\theta^{h}\right\|_{Q}^{2}+\left\|\varphi-\varphi^{h}\right\|_{W}^{2}\right) \\
& +\|\mathscr{M}\|\left(\left\|\theta-\theta^{h}\right\|_{Q}^{2}+\left\|u-u^{h}\right\|_{V}^{2}\right) \\
& +M_{k} c_{2}^{2}\left\|\theta-\theta^{h}\right\|_{Q}^{2} \\
& +L L_{k} c_{1} c_{2}\left(\left\|\theta-\theta^{h}\right\|_{Q}^{2}+\left\|u-u^{h}\right\|_{V}^{2}\right) \\
\leq & \alpha_{4}\left(\left\|u-u^{h}\right\|_{Q}^{2}+\left\|\varphi-\varphi^{h}\right\|_{W}^{2}+\left\|\theta-\theta^{h}\right\|_{Q}^{2}\right),
\end{aligned}
$$

where $\alpha_{4}$ is a positive constant. Keeping in mind the following $\alpha$-inequality $a b \leq \alpha a^{2}+(1 / \alpha) b^{2}$, it follows from (140)-(143) that if $m_{T}+\|\mathscr{P}\|+\|\mathscr{M}\|+L_{k} L+M_{k}<L^{*}$, then there exists a positive constant $c$ such that

$$
\begin{gathered}
\left\|u-u^{h}\right\|_{V}^{2}+\left\|\varphi-\varphi^{h}\right\|_{W}^{2}+\left\|\theta-\theta^{h}\right\|_{Q}^{2} \leq c\left(\left\|u-v^{h}\right\|_{V}^{2}\right. \\
\left.+\left\|u-v^{h}\right\|_{V}+\left\|\theta-\eta^{h}\right\|_{Q}^{2}+\left\|\varphi-\xi^{h}\right\|_{W}^{2}\right),
\end{gathered}
$$

and that finishes the proof of the Theorem 9.

As a result of the previous theorem, the following corollary is about the convergence order error estimates for the fully discrete approximations with the previous subspaces $V^{h}$, $W^{h}$, and $Q^{h}$. Let $\Pi^{h}$ be, as usual, the interpolation operator $\Pi^{h}: V \times W \times Q \rightarrow V^{h} \times W^{h} \times Q^{h}$. Using the standard finite element interpolation error estimates, we have the following approximations:

$$
\begin{gathered}
\left\|u-\Pi^{h} u\right\|_{V} \leq c h\|u\|_{H^{2}(\Omega)^{d}}, \\
\left\|\varphi-\Pi^{h} \varphi\right\|_{W} \leq \operatorname{ch}\|\varphi\|_{H^{2}(\Omega)}, \\
\left\|\theta-\Pi^{h} \theta\right\|_{Q} \leq \operatorname{ch}\|\theta\|_{H^{2}(\Omega)} .
\end{gathered}
$$

Corollary 10. Assume that conditions of Theorem 9 hold. Under the regularity conditions

$$
\begin{aligned}
& u \in H^{2}(\Omega)^{d}, \\
& \varphi \in H^{2}(\Omega), \\
& \theta \in H^{2}(\Omega),
\end{aligned}
$$

there exists positive constant $c>0$, independent of $h$, such that

$$
\left\|u-u^{h}\right\|_{V}^{2}+\left\|\varphi-\varphi^{h}\right\|_{W}^{2}+\left\|\theta-\theta^{h}\right\|_{Q}^{2} \leq c\left(h^{2}+h\right) .
$$

This corollary gives an estimation of the numerical errors of the problem $(P V)$ and its proof is based on the above approximation properties of the finite element spaces $V^{h}, W^{h}$, and $Q^{h}$.

Now, we investigate the particular case where $m_{T}=0$ and $1 \leq m_{n}<2$. Indeed, under some conditions on $\sigma_{\tau}$ and $c_{T}$, we can prove the following result.
Theorem 11. Let $m_{T}=0$ and $1 \leq m_{n}<2$. One assumes that, for some $s>3 / 2$, one has

$$
\begin{gathered}
\sigma_{\tau} \in H^{s-3 / 2}\left(\Gamma_{3}\right), \\
c_{T} \in H^{s-3 / 2}\left(\Gamma_{3}\right) .
\end{gathered}
$$

Then, the following error estimate can be obtained:

$$
\begin{aligned}
& \left\|u-u^{h}\right\|_{V}^{2}+\left\|\varphi-\varphi^{h}\right\|_{W}^{2}+\left\|\theta-\theta^{h}\right\|_{Q}^{2} \leq c\left\{\left\|u-v^{h}\right\|_{V}^{2}\right. \\
& +\left\|u-v^{h}\right\|_{H^{2-s}(\Omega)^{d}}+\left\|u-v^{h}\right\|_{L^{2}\left(\Gamma_{3}\right)^{d}}^{2} \\
& +\left\|u-v^{h}\right\|_{L^{2}\left(\Gamma_{3}\right)^{d}}+\left\|\theta-\eta^{h}\right\|_{Q}^{2}+\left\|\theta-\eta^{h}\right\|_{L^{2}\left(\Gamma_{3}\right)}^{2} \\
& \left.+\left\|\varphi-\xi^{h}\right\|_{W}^{2}\right\} .
\end{aligned}
$$

Proof. Since $m_{T}$ is zero, the functional $j_{T}$ becomes

$$
j_{T}^{0}(v)=\int_{\Gamma_{3}} c_{T}\left|v_{\tau}\right| d a .
$$

Then, the variational inequalities (46) and (123) can be written

$$
\begin{aligned}
& (\sigma, \varepsilon(v)-\varepsilon(u))_{\mathscr{H}}+j_{n}(u, v-u)+j_{T}^{0}(v) \\
& \quad-j_{T}^{0}(u) \geq(f, v-u)_{V}, \quad \forall v \in V, \\
& \left(\sigma^{h}, \varepsilon\left(v^{h}\right)-\varepsilon\left(u^{h}\right)\right)_{\mathscr{H}}+j_{n}\left(u^{h}, v^{h}-u^{h}\right)+j_{T}^{0}\left(v^{h}\right) \\
& \quad-j_{T}^{0}\left(u^{h}\right) \geq\left(f, v^{h}-u^{h}\right)_{V}, \quad \forall v^{h} \in V^{h} .
\end{aligned}
$$

We take $v=u^{h}$ in (151) to obtain

$$
\begin{aligned}
- & \left(\sigma, \varepsilon\left(u^{h}\right)-\varepsilon(u)\right)_{\mathscr{H}} \\
\leq & j_{n}\left(u, u^{h}-u\right)+j_{T}^{0}\left(u^{h}\right)-j_{T}^{0}(u) \\
& -\left(f, u^{h}-u\right)_{V},
\end{aligned}
$$

and from (152), we conclude

$$
\begin{aligned}
\left(\sigma^{h}, \varepsilon\left(u^{h}\right)-\varepsilon(u)\right)_{\mathscr{H}} & \\
\leq & \left(\sigma^{h}-\sigma, \varepsilon\left(v^{h}\right)-\varepsilon(u)\right)_{\mathscr{H}} \\
\quad+\left(\sigma, \varepsilon\left(v^{h}\right)-\varepsilon(u)\right)_{\mathscr{H}}+j_{n}\left(u^{h}, v^{h}-u^{h}\right) & \\
\quad+j_{T}^{0}\left(v^{h}\right)-j_{T}^{0}\left(u^{h}\right)-\left(f, v^{h}-u^{h}\right)_{V}, & \\
& \forall v^{h} \in V^{h} .
\end{aligned}
$$

Adding (153) and (154), we deduce

$$
\begin{aligned}
\left(\sigma^{h}-\sigma, \varepsilon\left(u^{h}\right)-\varepsilon(u)\right)_{\mathscr{H}} \\
\leq\left(\sigma^{h}-\sigma, \varepsilon\left(v^{h}\right)-\varepsilon(u)\right)_{\mathscr{H}} \\
\quad+\left(\sigma, \varepsilon\left(v^{h}\right)-\varepsilon(u)\right)_{\mathscr{H}}+j_{n}\left(u^{h}, v^{h}-u^{h}\right)
\end{aligned}
$$




$$
\begin{aligned}
& +j_{n}\left(u, u^{h}-u\right)+j_{T}^{0}\left(v^{h}\right)-j_{T}^{0}(u) \\
& -\left(f, v^{h}-u\right)_{V}, \quad \forall v^{h} \in V^{h} .
\end{aligned}
$$

We recall that $\sigma=\mathfrak{F} \varepsilon(u)-\mathscr{E}^{*} E(\varphi)-\mathscr{M} \theta$ and $\sigma^{h}=\mathfrak{F} \varepsilon\left(u^{h}\right)-$ $\mathscr{E}^{*} E\left(\varphi^{h}\right)-\mathscr{M} \theta^{h}$, then

$$
\begin{aligned}
(\mathfrak{F} \varepsilon & \left.(u)-\mathfrak{F} \varepsilon\left(u^{h}\right), \varepsilon\left(u^{h}\right)-\varepsilon(u)\right)_{\mathscr{H}} \\
& +\left(\mathscr{E}^{*} \nabla \varphi-\mathscr{E}^{*} \nabla \varphi^{h}, \varepsilon\left(u^{h}\right)-\varepsilon(u)\right)_{\mathscr{H}} \\
& -\left(\mathscr{M} \theta-\mathscr{M} \theta^{h}, \varepsilon\left(u^{h}\right)-\varepsilon(u)\right)_{\mathscr{H}} \\
\leq & \left(\sigma^{h}-\sigma, \varepsilon\left(v^{h}\right)-\varepsilon(u)\right)_{\mathscr{H}} \\
& +\left(\sigma, \varepsilon\left(v^{h}\right)-\varepsilon(u)\right)_{\mathscr{H}}+j_{n}\left(u^{h}, v^{h}-u^{h}\right) \\
& +j_{n}\left(u, u^{h}-u\right)+j_{T}^{0}\left(v^{h}\right)-j_{T}^{0}(u) \\
& -\left(f, v^{h}-u\right)_{V}, \quad \forall v^{h} \in V^{h} .
\end{aligned}
$$

Remembering (128) and (6), we replace $\left(\mathscr{E}^{*} \nabla \varphi\right.$ $\left.\mathscr{E}^{*} \nabla \varphi^{h}, \varepsilon\left(u^{h}\right)-\varepsilon(u)\right)_{\mathscr{C}}$ by its value. Hence

$$
\begin{aligned}
&\left(\mathfrak{F}_{\varepsilon}(u)-\mathfrak{F}^{\varepsilon}\left(u^{h}\right), \varepsilon\left(u^{h}\right)-\varepsilon(u)\right)_{\mathscr{H}} \\
& \quad+\left(\beta \nabla \varphi-\beta \nabla \varphi^{h}, \nabla \varphi-\nabla \varphi^{h}\right)_{H} \\
& \leq\left(\sigma^{h}-\sigma, \varepsilon\left(v^{h}\right)-\varepsilon(u)\right)_{\mathscr{C}} \\
&+\left(\sigma, \varepsilon\left(v^{h}\right)-\varepsilon(u)\right)_{\mathscr{H}}+j_{n}\left(u^{h}, v^{h}-u^{h}\right) \\
&+j_{n}\left(u, u^{h}-u\right)+j_{T}^{0}\left(v^{h}\right)-j_{T}^{0}(u) \\
&-\left(f, v^{h}-u\right)_{V}-\left(\beta \nabla \varphi-\beta \nabla \varphi^{h}, \nabla \xi^{h}-\nabla \varphi\right)_{H} \\
&+\left(\mathscr{P} \theta-\mathscr{P} \theta^{h}, \nabla \varphi-\nabla \varphi^{h}\right)_{H} \\
&+\left(\mathscr{P} \theta-\mathscr{P} \theta^{h}, \nabla \xi^{h}-\nabla \varphi\right)_{H} \\
&+\left(\mathscr{E} \varepsilon(u)-\mathscr{E} \varepsilon\left(u^{h}\right), \nabla \xi^{h}-\nabla \varphi\right)_{H} \\
&+\left(\mathscr{M} \theta-\mathscr{M} \theta^{h}, \varepsilon\left(u^{h}\right)-\varepsilon(u)\right)_{\mathscr{H}} .
\end{aligned}
$$

It follows from the sum of (134) and (157) that, for all $\left(v^{h}, \xi^{h}, \eta^{h}\right)$ of $V^{h} \times W^{h} \times Q^{h}$, we have

$$
\begin{aligned}
& \left(\mathfrak{F} \varepsilon(u)-\mathfrak{F} \varepsilon\left(u^{h}\right), \varepsilon\left(u^{h}\right)-\varepsilon(u)\right)_{\mathscr{H}} \\
& \quad+\left(\beta \nabla \varphi-\beta \nabla \varphi^{h}, \nabla \varphi-\nabla \varphi^{h}\right)_{H} \\
& +\left(\mathscr{K} \nabla \theta-\mathscr{K} \nabla \theta^{h}, \nabla \theta-\nabla \theta^{h}\right)_{H} \leq\left(\sigma^{h}-\sigma,\right. \\
& \left.\quad \varepsilon\left(v^{h}\right)-\varepsilon(u)\right)_{\mathscr{H}}+\left(\sigma, \varepsilon\left(v^{h}\right)-\varepsilon(u)\right)_{\mathscr{H}}+j_{n}\left(u^{h},\right. \\
& \left.v^{h}-u^{h}\right)+j_{n}\left(u, u^{h}-u\right)+j_{T}^{0}\left(v^{h}\right)-j_{T}^{0}(u)-(f,
\end{aligned}
$$

$$
\begin{aligned}
& \left.v^{h}-u\right)_{V}-\left(\beta \nabla \varphi-\beta \nabla \varphi^{h}, \nabla \xi^{h}-\nabla \varphi\right)_{H}+(\mathscr{P} \theta \\
& \left.-\mathscr{P} \theta^{h}, \nabla \varphi-\nabla \varphi^{h}\right)_{H}+\left(\mathscr{P} \theta-\mathscr{P} \theta^{h}, \nabla \xi^{h}-\nabla \varphi\right)_{H} \\
& +\left(\mathscr{E} \varepsilon(u)-\mathscr{E} \varepsilon\left(u^{h}\right), \nabla \xi^{h}-\nabla \varphi\right)_{H}+(\mathscr{M} \theta \\
& \left.-\mathscr{M} \theta^{h}, \varepsilon\left(u^{h}\right)-\varepsilon(u)\right)_{\mathscr{H}}+\left(\mathscr{K} \nabla \theta-\mathscr{K} \nabla \theta^{h}, \nabla \theta\right. \\
& \left.-\nabla \eta^{h}\right)_{H}-\int_{\Gamma_{3}}\left[k\left(u_{v}^{h}-g\right) \phi_{L}\left(\theta^{h}-\theta_{F}\right)\right. \\
& \left.-k\left(u_{v}-g\right) \phi_{L}\left(\theta-\theta_{F}\right)\right]\left(\theta-\theta^{h}\right) d a \\
& +\int_{\Gamma_{3}}\left[k\left(u_{v}^{h}-g\right) \phi_{L}\left(\theta^{h}-\theta_{F}\right)\right. \\
& \left.-k\left(u_{v}-g\right) \phi_{L}\left(\theta-\theta_{F}\right)\right]\left(\theta-\eta^{h}\right) d a .
\end{aligned}
$$

To simplify the calculations, let us consider the following quantities:

$$
\begin{aligned}
\widetilde{I}_{1} & =\left(\sigma^{h}-\sigma, \varepsilon\left(v^{h}\right)-\varepsilon(u)\right)_{\mathscr{H}}-\left(\beta \nabla \varphi-\beta \nabla \varphi^{h}, \nabla \xi^{h}\right. \\
& -\nabla \varphi)_{H}+\left(\mathscr{P} \theta-\mathscr{P} \theta^{h}, \nabla \xi^{h}-\nabla \varphi\right)_{H}+(\mathscr{E} \varepsilon(u) \\
& \left.-\mathscr{E} \varepsilon\left(u^{h}\right), \nabla \xi^{h}-\nabla \varphi\right)_{H}+\left(\mathscr{K} \nabla \theta-\mathscr{K} \nabla \theta^{h}, \nabla \theta\right. \\
& \left.-\nabla \eta^{h}\right)_{H}+\int_{\Gamma_{3}}\left[k\left(u_{v}^{h}-g\right) \phi_{L}\left(\theta^{h}-\theta_{F}\right)\right. \\
& \left.-k\left(u_{v}-g\right) \phi_{L}\left(\theta-\theta_{F}\right)\right]\left(\theta-\eta^{h}\right) d a, \\
\widetilde{I}_{2} & =\left(\sigma, \varepsilon\left(v^{h}\right)-\varepsilon(u)\right)_{\mathscr{H}}+j_{T}^{0}\left(v^{h}\right)-j_{T}^{0}(u)-\left(f, v^{h}\right. \\
& -u)_{V}, \\
\widetilde{I}_{3} & =j_{n}\left(u^{h}, v^{h}-u^{h}\right)+j_{n}\left(u, u^{h}-u\right)=\int_{\Gamma_{3}} c_{n}\left(u_{v}^{h}\right. \\
& -g)_{+}^{m_{n}}\left(v_{v}^{h}-u_{v}^{h}\right) d a+\int_{\Gamma_{3}} c_{n}\left(u_{v}-g\right)_{+}^{m_{n}}\left(u_{v}^{h}\right. \\
& \left.-u_{v}\right) d a, \\
\widetilde{I}_{4} & =\left(\mathscr{P} \theta-\mathscr{P} \theta^{h}, \nabla \varphi-\nabla \varphi^{h}\right)_{H}+\left(\mathscr{M} \theta-\mathscr{M} \theta^{h},\right. \\
& \left.\varepsilon\left(u^{h}\right)-\varepsilon(u)\right)_{\mathscr{H}}-\int_{\Gamma_{3}}\left[k\left(u_{v}^{h}-g\right) \phi_{L}\left(\theta^{h}-\theta_{F}\right)\right. \\
& \left.-k\left(u_{v}-g\right) \phi_{L}\left(\theta-\theta_{F}\right)\right]\left(\theta-\theta^{h}\right) d a .
\end{aligned}
$$

Taking $\theta^{h}-\theta_{F}=\left(\theta^{h}-\theta\right)+\left(\theta-\theta_{F}\right)$ in the integral term of (159), it comes from (26), (30), and the assumptions $\left(\mathrm{h}_{1}\right)-\left(\mathrm{h}_{4}\right)$ that

$$
\begin{gathered}
\widetilde{I}_{1} \leq\left\|\sigma^{h}-\sigma\right\|\left\|v^{h}-u\right\|_{V}-\|\beta\|\left\|\varphi-\varphi^{h}\right\|_{W}\left\|\xi^{h}-\varphi\right\|_{W} \\
+\|\mathscr{P}\|\left\|\theta-\theta^{h}\right\|_{Q}\left\|\xi^{h}-\varphi\right\|_{W}
\end{gathered}
$$




$$
\begin{aligned}
& +\|\mathscr{E}\|\left\|u-u^{h}\right\|_{V}\left\|\xi^{h}-\varphi\right\|_{W} \\
& +\|\mathscr{K}\|\left\|\theta-\theta^{h}\right\|_{Q}\left\|\theta-\eta^{h}\right\|_{Q} \\
& +M_{k} c_{2}\left\|\theta-\theta_{h}\right\|_{Q}\left\|\theta-\eta^{h}\right\|_{L^{2}\left(\Gamma_{3}\right)} \\
& +L L_{k} c_{0}\left\|u-u^{h}\right\|_{V}\left\|\theta-\eta^{h}\right\|_{L^{2}\left(\Gamma_{3}\right)} .
\end{aligned}
$$

Using the Green formula and recalling (2), (9), (10), (15), and (16), we obtain

$$
\begin{aligned}
I_{2}^{\prime}= & \int_{\Omega} \sigma\left(\varepsilon\left(v^{h}\right)-\varepsilon(u)\right) d a+j_{T}^{0}\left(v^{h}\right)-j_{T}^{0}(u) \\
& -\left(f, v^{h}-u\right)_{V} \\
= & \int_{\Gamma} \sigma v\left(v^{h}-u\right) d a-\int_{\Omega} \operatorname{Div}(\sigma)\left(v^{h}-u\right) d x \\
& +j_{T}^{0}\left(v^{h}\right)-j_{T}^{0}(u)-\left(f, v^{h}-u\right)_{V} \\
= & \int_{\Gamma_{2}} f_{2}\left(v^{h}-u\right) d a+\int_{\Gamma_{3}} \sigma_{v}\left(v_{v}^{h}-u_{v}\right) d a \\
& +\int_{\Gamma_{3}} \sigma_{\tau}\left(v_{\tau}^{h}-u_{\tau}\right) d a+\int_{\Omega} f_{0}\left(v^{h}-u\right) d x \\
& +j_{T}^{0}\left(v^{h}\right)-j_{T}^{0}(u)-\left(f, v^{h}-u\right)_{V}
\end{aligned}
$$

and since $\left(f, v^{h}-u\right)_{V}=\int_{\Omega} f_{0}\left(v^{h}-u\right) d x+\int_{\Gamma_{2}} f_{2}\left(v^{h}-u\right) d a$, we have

$$
\begin{aligned}
\widetilde{I}_{2}= & \int_{\Gamma_{3}} \sigma_{\nu}\left(v_{\nu}^{h}-u_{v}\right) d a+\int_{\Gamma_{3}} \sigma_{\tau}\left(v_{\tau}^{h}-u_{\tau}\right) d a \\
& +j_{T}^{0}\left(v^{h}\right)-j_{T}^{0}(u) \\
= & \int_{\Gamma_{3}}-c_{n}\left(u_{\nu}-g\right)_{+}^{m_{n}}\left(v_{\nu}^{h}-u_{\nu}\right) d a \\
& +\int_{\Gamma_{3}} \sigma_{\tau}\left(v_{\tau}^{h}-u_{\tau}\right) d a+\int_{\Gamma_{3}} c_{T}\left(\left|v_{\tau}^{h}\right|-\left|u_{\tau}\right|\right) d a .
\end{aligned}
$$

Hence

$$
\begin{aligned}
\widetilde{I}_{2} & \leq\left\|c_{n}\left(u_{v}-g\right)_{+}^{m_{n}}\right\|_{L^{2}\left(\Gamma_{3}\right)}\left\|v_{\nu}^{h}-u_{\nu}\right\|_{L^{2}\left(\Gamma_{3}\right)} \\
& +\left(\left\|\sigma_{\tau}\right\|_{H^{s-3 / 2}\left(\Gamma_{3}\right)}+\left\|c_{T}\right\|_{H^{s-3 / 2}\left(\Gamma_{3}\right)}\right)\left\|v_{\tau}^{h}-u_{\tau}\right\|_{H^{-s+3 / 2}\left(\Gamma_{3}\right)} \\
& \leq\left\|c_{n}\left(u_{v}-g\right)_{+}^{m_{n}}\right\|_{L^{2}\left(\Gamma_{3}\right)}\left\|v^{h}-u\right\|_{L^{2}\left(\Gamma_{3}\right)^{d}} \\
& +\left(\left\|\sigma_{\tau}\right\|_{H^{s-3 / 2}\left(\Gamma_{3}\right)}+\left\|c_{T}\right\|_{H^{s-3 / 2}\left(\Gamma_{3}\right)}\right)\left\|v^{h}-u\right\|_{H^{-s+2}(\Omega)^{d}} .
\end{aligned}
$$

Moreover, taking $v_{v}^{h}-u_{v}^{h}=\left(v_{v}^{h}-u_{v}\right)+\left(u_{v}-u_{v}^{h}\right)$ in the first integral of (161) and using the Lemma 2, we have

$$
\widetilde{I}_{3}
$$$$
=\int_{\Gamma_{3}} c_{n}\left(u_{\nu}^{h}-g\right)_{+}^{m_{n}}\left(v_{\nu}^{h}-u_{\nu}\right) d a
$$

$$
\begin{aligned}
& +\int_{\Gamma_{3}} c_{n}\left(\left(u_{v}^{h}-g\right)_{+}^{m_{n}}-\left(u_{v}-g\right)_{+}^{m_{n}}\right)\left(u_{v}-u_{\nu}^{h}\right) d a \\
\leq & \int_{\Gamma_{3}} c_{n}\left(u_{v}^{h}-g\right)_{+}^{m_{n}}\left(v_{\nu}^{h}-u_{v}\right) d a
\end{aligned}
$$

and then we conclude

$$
\begin{aligned}
& \widetilde{I}_{3} \leq\left\|c_{n}\left(u_{v}^{h}-g\right)_{+}^{m_{n}}\right\|_{L^{2}\left(\Gamma_{3}\right)}\left\|v_{\nu}^{h}-u_{\nu}\right\|_{L^{2}\left(\Gamma_{3}\right)} \leq\left\|c_{n}\right\|_{L^{\infty}\left(\Gamma_{3}\right)} \\
& \cdot\left\|\left(u_{\nu}^{h}-g\right)_{+}^{m_{n}}\right\|_{L^{2}\left(\Gamma_{3}\right)}\left\|v_{v}^{h}-u_{\nu}\right\|_{L^{2}\left(\Gamma_{3}\right)} \\
& \leq\left\|c_{n}\right\|_{L^{\infty}\left(\Gamma_{3}\right)}\left\|u_{v}^{h}-g\right\|_{L^{2 m_{n}\left(\Gamma_{3}\right)}}\left\|v_{\nu}^{h}-u_{\nu}\right\|_{L^{2}\left(\Gamma_{3}\right)} \\
& \leq\left\|c_{n}\right\|_{L^{\infty}\left(\Gamma_{3}\right)} \\
& \cdot\left(\left\|u^{h}-u\right\|_{L^{2 m_{n}\left(\Gamma_{3}\right)^{d}}}+\|u\|_{L^{2 m_{n}\left(\Gamma_{3}\right)^{d}}}+\|g\|_{L^{2 m_{n}\left(\Gamma_{3}\right)}}\right) \\
& \cdot\left\|v_{\nu}^{h}-u_{\nu}\right\|_{L^{2}\left(\Gamma_{3}\right)} \leq\left\|c_{n}\right\|_{L^{\infty}\left(\Gamma_{3}\right)} \\
& \cdot\left(N_{2 m_{n}}\left\|u^{h}-u\right\|_{V}+N_{2 m_{n}}\|u\|_{V}+\|g\|_{L^{2 m_{n}\left(\Gamma_{3}\right)}}\right) \\
& \cdot\left\|v^{h}-u\right\|_{L^{2}\left(\Gamma_{3}\right)^{d}} \text {. }
\end{aligned}
$$

However, we take $\theta^{h}-\theta_{F}=\left(\theta^{h}-\theta\right)+\left(\theta-\theta_{F}\right)$ in the integral term of (162) and we use conditions $\left(\mathrm{h}_{2}\right)-\left(\mathrm{h}_{4}\right)$ and (26), (30) to find

$$
\begin{aligned}
\widetilde{I}_{4} \leq\|\mathscr{P}\|\left\|\theta-\theta^{h}\right\|_{Q}\left\|\varphi-\varphi^{h}\right\|_{W}+\|\mathscr{M}\|\left\|\theta-\theta^{h}\right\|_{Q} \\
\cdot\left\|u^{h}-u\right\|_{V}+M_{k} c_{2}^{2}\left\|\theta-\theta^{h}\right\|_{Q}^{2} \\
+L L_{k} \mathcal{c}_{0} \mathcal{c}_{2}\left\|u^{h}-u\right\|_{V}\left\|\theta-\theta^{h}\right\|_{Q} \\
\leq \widetilde{C}\left(\|\mathscr{P}\|+\|\mathscr{M}\|+M_{k}+L L_{k}\right) \\
\cdot\left(\left\|\theta-\theta^{h}\right\|_{Q}^{2}+\left\|\varphi-\varphi^{h}\right\|_{W}^{2}+\left\|u^{h}-u\right\|_{V}^{2}\right),
\end{aligned}
$$

where $\widetilde{C}=\max \left(1, c_{2}^{2}, c_{0} c_{2}\right)$. If we suppose $m_{T}+\|\mathscr{P}\|+\|\mathscr{M}\|+$ $M_{k}+L_{k} L<L^{*}$ with

$$
L^{*}=\frac{\max \{R, \widetilde{C}\}}{\min \left\{m_{\mathscr{F}}, m_{\beta}, m_{\mathscr{K}}\right\}},
$$

then

$$
\widetilde{C}\left(\|\mathscr{P}\|+\|\mathscr{M}\|+M_{k}+L L_{k}\right)<1 .
$$

Keeping in mind $\left(\mathrm{h}_{1}\right)$ and (158), (159), (160), (161), (162), (163), (166), (168), (169), and (171) we apply several times the 
$\alpha$-inequality to conclude that there exists a constant $c>0$ such that, for all $\left(u^{h}, \xi^{h}, \eta^{h}\right)$ of $V^{h} \times W^{h} \times Q^{h}$, we have

$$
\begin{aligned}
& \left\|u-u^{h}\right\|_{V}^{2}+\left\|\varphi-\varphi^{h}\right\|_{W}^{2}+\left\|\theta-\theta^{h}\right\|_{Q}^{2} \leq c\left(\left\|u-v^{h}\right\|_{V}^{2}\right. \\
& +\left\|u-v^{h}\right\|_{H^{-s+2}(\Omega)^{d}}+\left\|u-v^{h}\right\|_{L^{2}\left(\Gamma_{3}\right)^{d}}^{2} \\
& +\left\|u-v^{h}\right\|_{L^{2}\left(\Gamma_{3}\right)^{d}}+\left\|\theta-\eta^{h}\right\|_{Q}^{2}+\left\|\theta-\eta^{h}\right\|_{L^{2}\left(\Gamma_{3}\right)}^{2} \\
& \left.+\left\|\varphi-\xi^{h}\right\|_{W}^{2}\right) .
\end{aligned}
$$

\section{Conclusion}

In this work, we presented a model for the static process of frictional contact between a piezoelectric body and an electrically thermally conductive foundation. The constitutive relation of the material is assumed to be thermo-electroelastic. The contact was modeled with the normal compliance condition and the associated Coulomb's friction law, including the electrical and thermal conductivity conditions. The existence of the unique weak solution for the problem was established by using arguments from the theory of variational inequalities and a fixed point theorem. A discrete scheme by finite element method was used to approach the problem and an optimal order error estimate was derived. A numerical validation of the convergence result included in this method will be provided in a forthcoming paper.

\section{Conflicts of Interest}

The authors declare that there are no conflicts of interest regarding the publication of this paper.

\section{References}

[1] M. Aouadi, "Generalized thermo-piezoelectric problems with temperature-dependent properties," International Journal of Solids and Structures, vol. 43, no. 21, pp. 6347-6358, 2006.

[2] D. S. Chandrasekharaiah, "A generalized linear thermoelasticity theory for piezoelectric media," Acta Mechanica, vol. 71, no. 1-4, pp. 39-49, 1988.

[3] W. Nowacki, "Some general theorems of thermo-piezoelectricity," Journal of Thermal Stresses, vol. 1, no. 2, pp. 171-182, 1978.

[4] H. F. Tiersten, "On the nonlinear equations of thermoelectroelasticity," International Journal of Engineering Science, vol. 9, pp. 587-604, 1971.

[5] E.-H. Essoufi, E.-H. Benkhira, and R. Fakhar, "Analysis and numerical approximation of an electro-elastic frictional contact problem," Advances in Applied Mathematics and Mechanics, vol. 2, no. 3, pp. 355-378, 2010.

[6] P. Bisegna, F. Lebon, and F. Maceri, "The unilateral frictional contact of a piezoelectric body with a rigid support," in Contact Mechanics, vol. 103, pp. 347-354, Kluwer, Dordrecht, The Netherlands, 2002.

[7] G. Duvaut and J.-L. Lions, Inequalities in Mechanics and Physics, Springer, Berlin, Germany, 1976.
[8] M. Sofonea and A. Matei, Mathematical Models in Contact Mechanics, Lecture Note, Series 398, Cambridge University Press, Cambridge, UK, 2012.

[9] L.-E. Andersson, A. Klarbring, J. R. Barber, and M. Ciavarella, "On the existence and uniqueness of steady state solutions in thermoelastic contact with frictional heating," Proceedings of the Royal Society A Mathematical, Physical and Engineering Sciences, vol. 461, no. 2057, pp. 1261-1282, 2005.

[10] G. Duvaut, "Free boundary problem connected with thermoelasticity and unilateral contact," in Free boundary problems, pp. 217-236, 1980.

[11] M. A. Ezzat and E. S. Awad, "Constitutive relations, uniqueness of solution, and thermal shock application in the linear theory of micropolar generalized thermoelasticity involving two temperatures," Journal of Thermal Stresses, vol. 33, no. 3, pp. 226$250,2010$.

[12] A. S. El-Karamany and M. A. Ezzat, "On the boundary integral formulation of thermo-viscoelasticity theory," International Journal of Engineering Science, vol. 40, no. 17, pp. 1943-1956, 2002.

[13] Z. Lerguet, M. Shillor, and M. Sofonea, "A frictional contact problem for an electro-viscoelastic body," Electronic Journal of Differential Equations, vol. 170, pp. 1-16, 2007.

[14] H. Benaissa, E.-H. Essoufi, and R. Fakhar, "Existence results for unilateral contact problem with friction of thermo-electroelasticity," Applied Mathematics and Mechanics-English Edition, vol. 36, no. 7, pp. 911-926, 2015.

[15] H. Benaissa, E. L.-H. Essoufi, and R. Fakhar, "Analysis of a Signorini problem with nonlocal friction in thermopiezoelectricity," Glasnik Matematički, vol. 51, no. 2, pp. 391-411, 2016.

[16] H. Benaissa, E.-H. Essoufi, and R. Fakhar, "Variational analysis of thermo-piezoelectric contact problem with friction," Journal of Advanced Research in Applied Mathematics, vol. 7, no. 2, pp. 52-75, 2015.

[17] A. Klarbring, A. Mikelic, and M. Shillor, "On friction problems with normal compliance," Nonlinear Analysis: Theory, Methods \& Applications, vol. 13, no. 8, pp. 935-955, 1989.

[18] J. A. C. Martins and J. T. Oden, "Existence and uniqueness results for dynamic contact problems with nonlineaire normal and friction interface laws," Nonlineaire Analysis. Theory, Methods \& Applications, vol. 11, no. 3, pp. 407-428, 1986.

[19] P. Hild, "Two results on solution uniqueness and multiplicity for the linear elastic friction problem with normal compliance," Nonlinear Analysis: Theory, Methods \& Applications, vol. 71, no. 11, pp. 5560-5571, 2009.

[20] J. Nečas and I. Hlaváček, Mathematical Theory of Elastic and Elasto-Plastic Bodies: An Introduction, Elsevier Scientific, Amsterdam, The Netherlands, 1981.

[21] R. A. Adams, Sobolev Spaces, Academic Press, NY, USA, 1975.

[22] S. a. Migórski, A. Ochal, and M. Sofonea, Nonlinear inclusions and hemivariational inequalities: Models and analysis of contact problems, Springer, NY, USA, 2014.

[23] G. H. Hardy, J. E. Littlewood, and G. Pólya, Inequalities, Cambridge University Press, London, UK, 2nd edition, 1952. 


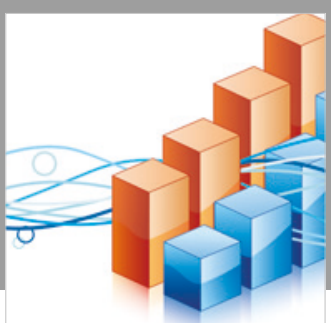

Advances in

Operations Research

\section{-n-m}
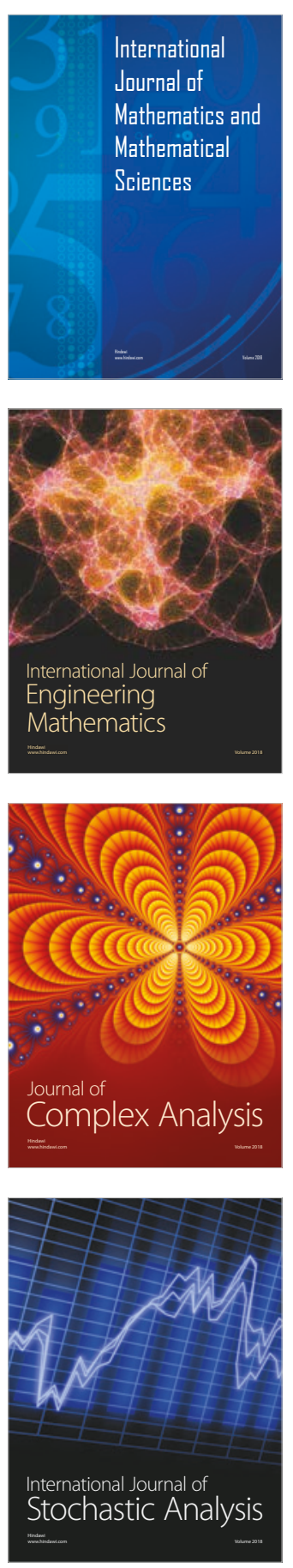
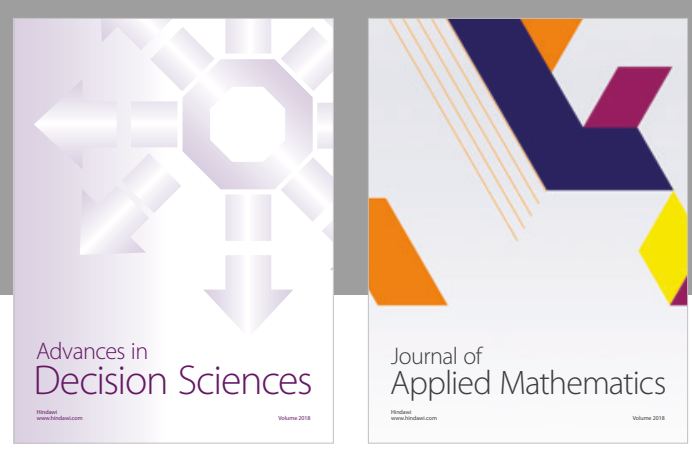

Journal of

Applied Mathematics
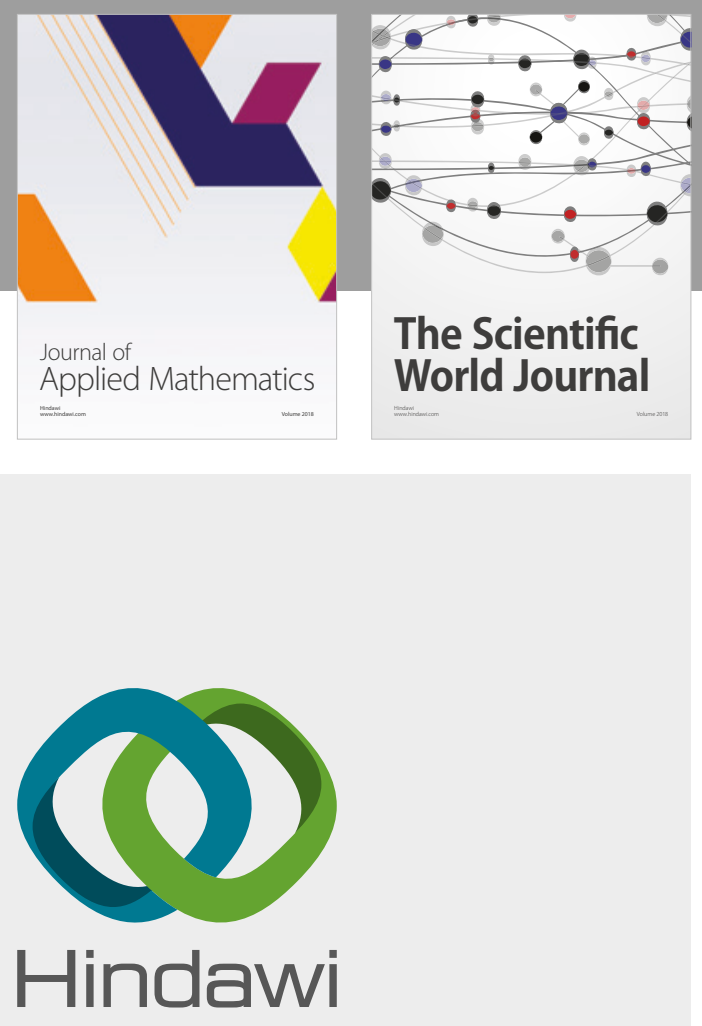

Submit your manuscripts at

www.hindawi.com

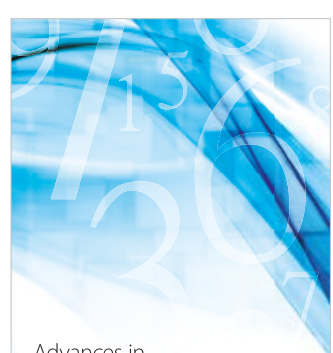

Advances in
Numerical Analysis
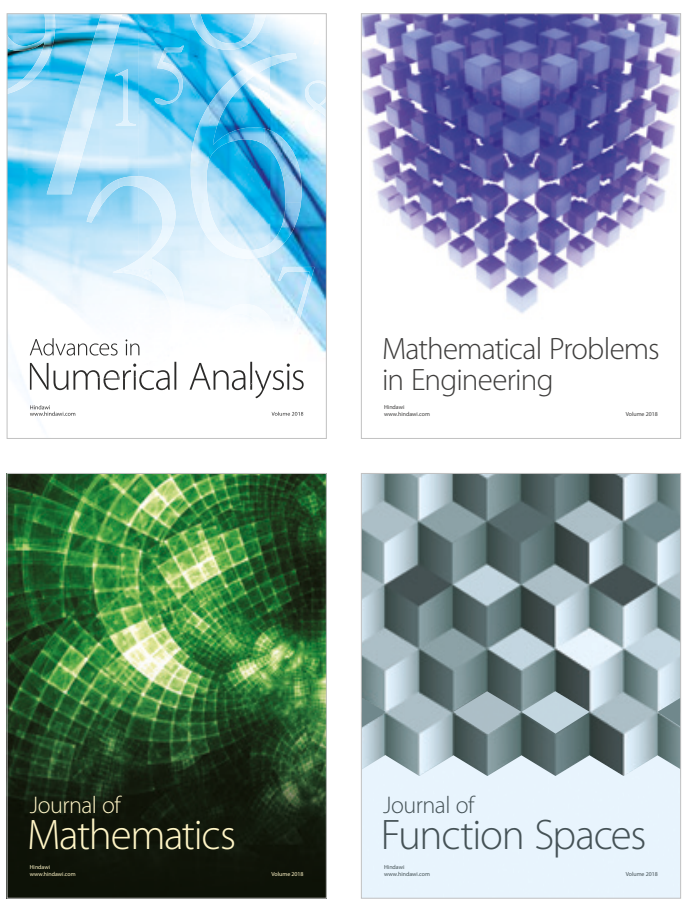

Mathematical Problems in Engineering

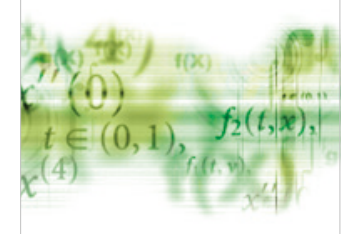

International Journal of

Differential Equations

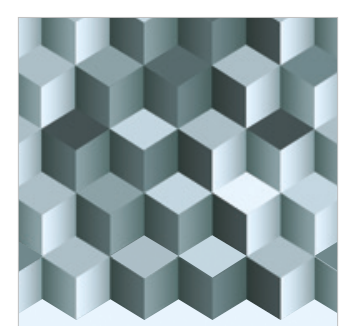

Journal of

Function Spaces

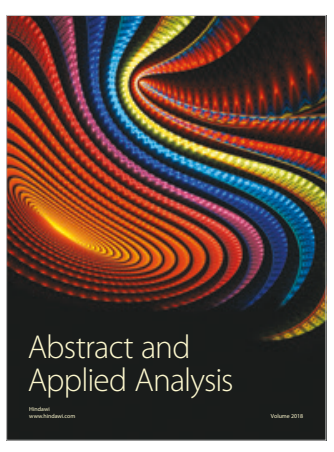

The Scientific

World Journal

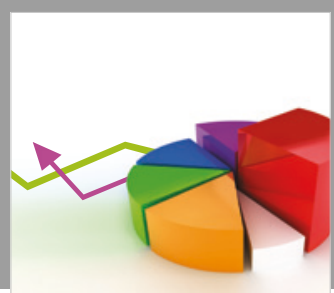

Journal of

Probability and Statistics
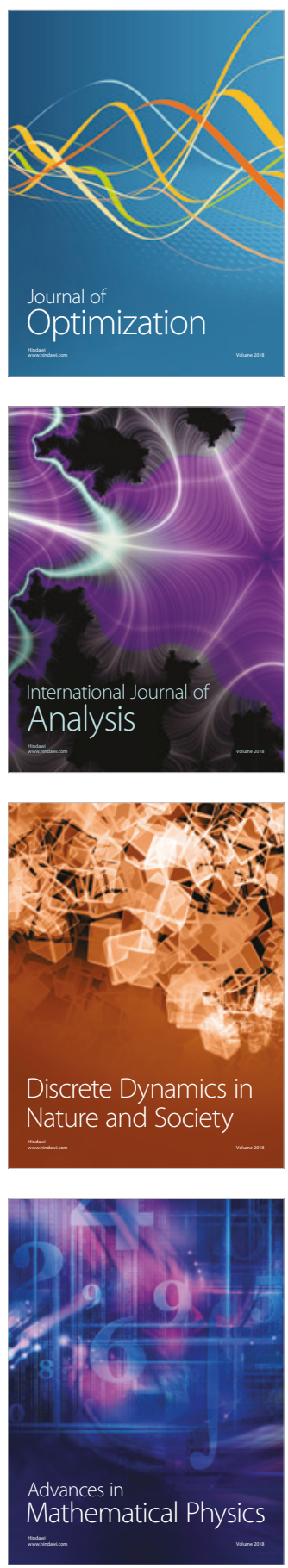